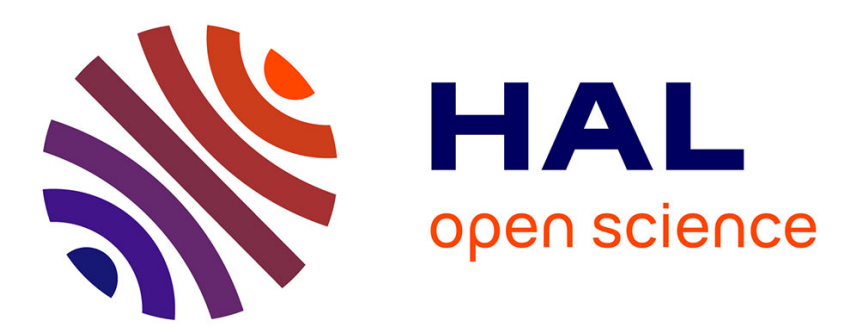

\title{
Numerical stability analysis of a vortex ring with swirl
} Yuji Hattori, Francisco J. Blanco-Rodríguez, Stéphane Le Dizès

\section{To cite this version:}

Yuji Hattori, Francisco J. Blanco-Rodríguez, Stéphane Le Dizès. Numerical stability analysis of a vortex ring with swirl. Journal of Fluid Mechanics, 2019, 878, pp.5-36. 10.1017/jfm.2019.621 . hal02321937

\section{HAL Id: hal-02321937 \\ https://hal.science/hal-02321937}

Submitted on 29 Jan 2020

HAL is a multi-disciplinary open access archive for the deposit and dissemination of scientific research documents, whether they are published or not. The documents may come from teaching and research institutions in France or abroad, or from public or private research centers.
L'archive ouverte pluridisciplinaire HAL, est destinée au dépôt et à la diffusion de documents scientifiques de niveau recherche, publiés ou non, émanant des établissements d'enseignement et de recherche français ou étrangers, des laboratoires publics ou privés. 


\title{
Numerical Stability Analysis of a Vortex Ring with Swirl
}

\section{Yuji Hattori, ${ }^{1} \dagger$ Francisco J. Blanco-Rodríguez ${ }^{2,3}$ and Stéphane Le Dizès $^{2}$}

\author{
${ }^{1}$ Institute of Fluid Science, Tohoku University, Sendai 980-8577, Japan \\ ${ }^{2}$ Aix Marseille Université, CNRS, Centrale Marseille, IRPHE, UMR 7342, 13384 Marseille, \\ France \\ ${ }^{3}$ Área de Mecánica de fluidos, Departamento de Ingeniería Aeroespacial y Mecánica de fluidos, \\ Universidad de Sevilla, Avda. de los Descubrimientos, s/n, 41092 Sevilla, Spain
}

\section{(Received xx; revised xx; accepted xx)}

The linear instability of a vortex ring with swirl with Gaussian distributions of azimuthal vorticity and velocity in its core is studied by direct numerical simulation (DNS). The numerical study is carried out in two steps: first, an axisymmetric simulation of the Navier-Stokes equations is performed to obtain the quasi-steady state that forms a base flow; then, the equations are linearized around this base flow and integrated for a sufficiently long time to obtain the characteristics of the most unstable mode. It is shown that the vortex rings are subjected to the curvature instability as predicted analytically by Blanco-Rodríguez \& Le Dizès (2017). Both the structure and the growth rate of the unstable modes obtained numerically are in good agreement with the analytical results. However, a small overestimation (e.g. $22 \%$ for a curvature instability mode) by the theory of the numerical growth rate is found for some instability modes. This is most likely due to evaluation of the critical layer damping which is performed for the waves on axisymmetric line vortices in the analysis. The actual position of the critical layer is affected by deformation of the core due to the curvature effect; as a result, the damping rate changes since it is sensitive to the position of the critical layer. Competition between the curvature and elliptic instabilities is also investigated. Without swirl, only the elliptic instability is observed in agreement with previous numerical and experimental results. In the presence of swirl, sharp bands of both curvature and elliptic instabilities are obtained for $\varepsilon=a / R=0.1$, where $a$ is the vortex core radius and $R$ the ring radius, while the elliptic instability dominates for $\varepsilon=0.18$. New types of instability mode are also obtained: a special curvature mode composed of three waves is observed and spiral modes that do not seem to be related to any wave resonance. The curvature instability is also confirmed by direct numerical simulation of the full Navier-Stokes equations. Weakly nonlinear saturation and subsequent decay of the curvature instability are also observed.

Key words: Authors should not enter keywords on the manuscript, as these must be chosen by the author during the online submission process and will then be added during the typesetting process (see http://journals.cambridge.org/data/relatedlink/jfmkeywords.pdf for the full list)

$\dagger$ Email address for correspondence: hattori@fmail.ifs.tohoku.ac.jp 


\section{Introduction}

Vortex rings are one of the fundamental vortices which appear in various flows. They are formed when fluid is ejected into a quiescent fluid from a circular hole whose size ranges from human lips to volcanoes. They also appear as coherent structures in a circular jet. An important feature of vortex rings is that vortex tubes have curvature, which distinguishes them from line vortices. Curvature has significant effects on the dynamics of a vortex ring. A vortex ring propagates owing to self-induced velocity due to curvature (Saffman 1992; Fukumoto \& Moffatt 2000). More importantly, curvature deforms the core of a vortex ring to give rise to short-wave instabilities (Fukumoto \& Hattori 2005; Hattori \& Fukumoto 2003).

Currently, two types of short-wave instabilities are known for vortex rings: elliptic instability and curvature instability. The elliptic instability of a vortex ring was established about four decades ago; Widnall \& Tsai (1977) showed that the strain induced on the core of a vortex ring by itself resonates with two neutral waves to give rise to an instability. The essential mechanism had been understood for a strained line vortex by Moore \& Saffman (1975) and Tsai \& Widnall (1976). Their theoretical results were in reasonable agreement with the experimental results of Widnall \& Sullivan (1973), which supports that the observed growth of bending waves is due to the elliptic instability. On the other hand, the curvature instability was first found theoretically by Fukumoto \& Hattori (2005) and Hattori \& Fukumoto (2003); it is caused by the dipolar field which is selfinduced on the core of a vortex ring and resonates with two neutral waves as in the case of elliptic instability. One of the important differences between the elliptic and curvature instabilities is the set of azimuthal wavenumbers of the coupled waves: $(m, m+2)$, where $m$ is an integer, in the elliptic instability and $(m, m+1)$ in the curvature instability. The curvature instability has been shown to exist theoretically also for Hill's spherical vortices (Hattori \& Hijiya 2010) and helical vortices (Hattori \& Fukumoto 2009, 2010, 2011, 2012, 2014).

It should be pointed out that the above results on the curvature instability and the results of Widnall \& Tsai (1977) are obtained for Kelvin's vortex ring for which vorticity is constant inside the core at the leading-order of perturbation expansion and vanishes outside the core. However, the vortex rings observed in the experiments usually have smooth vorticity distributions which are often approximated by Gaussian distributions. Recently, Blanco-Rodríguez \& Le Dizès (2016) and Blanco-Rodríguez \& Le Dizès (2017) have shown theoretically that vortex rings which have Gaussian distributions of azimuthal vorticity and velocity at the leading order are also subjected to elliptic and curvature instabilities. They found that some important features of the instabilities are quite different from those of Kelvin's vortex ring. In fact, characteristics of the waves on the Gaussian vortices change dramatically when there exists a critical layer whose radius satisfies

$$
\omega-k u_{\phi}^{(0)}(r)-m \Omega^{(0)}(r)=0,
$$

where $\omega$ is the oscillation frequency, $k$ and $m$ are the azimuthal and circumferential wavenumbers, respectively, and $u_{\phi}^{(0)}(r)$ and $\Omega^{(0)}(r)$ are the azimuthal velocity and angular velocity distributions of the vortices, respectively; some waves are damped with non-vanishing decay rate in the inviscid limit and the dispersion curves are distorted (Le Dizès 2004; Fabre, Sipp \& Jacquin 2006). It turns out that the curvature instability is much more affected by the presence of critical layers than the elliptic instability. However, there do exist unstable modes of the curvature instability of which growth rates can be comparable to those of the elliptic instability in the presence of azimuthal velocity. 

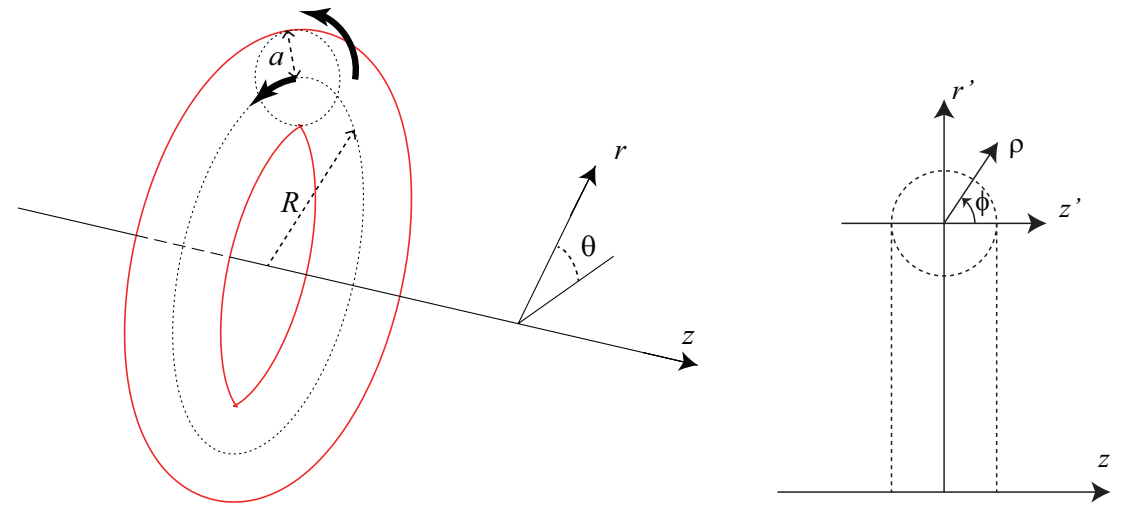

Figure 1. Flow model and coordinate system.

Experimental results on the instability of a vortex ring (Maxworthy 1972; Widnall \& Sullivan 1973; Maxworthy 1977; Dazin, Dupont \& Stanislas 2006a,b; Thompson, Leweke \& Hourigan 2007; Gan, Nickels \& Dawson 2011) have not been fully understood in spite of many efforts; one of the reasons for this is that it is not always easy to measure detailed structures of destabilized vortex rings. Numerical results have revealed some features of the elliptic instability; a map of unstable modes has been obtained recently by Gargan-Shingles, Rudman \& Ryan (2016) for vortex rings with swirl, where swirl implies the azimuthal or toroidal component of velocity in the present paper. See also Mao \& Hussain (2017) for transient growth and Shariff, Verzicco \& Orlandi (1994) for an early pioneering work. However, clear evidence of the curvature instability has not been observed in experiments or numerical simulations as far as the authors know.

In the present paper, we study the linear instability of vortex rings by direct numerical simulation (DNS); Gaussian vortex rings which have swirl in general are considered as a base flow. Our first aim is to show that curvature instability exists in reality by DNS. After confirming its existence the growth rates of the unstable eigenmodes are evaluated and compared to the theoretical results. The structures of the unstable eigenmodes are also revealed. Nonlinear effects are also investigated to observe whether weakly nonlinear saturation occurs or not.

The paper is organized as follows. In $\S 2$ we describe the numerical procedure. In $\S 3$ some important features of numerically obtained base flow are presented. The linear stability results are shown in $\S 4$, while nonlinear effects are studied in $\S 5$. We conclude in $\S 6$.

\section{Numerical procedure}

\subsection{Outline}

We consider the instability of a vortex ring in an incompressible viscous fluid (figure $1)$; the vortex ring has swirl in general. We take a cylindrical coordinate system $(r, \theta, z)$, where the axis of symmetry of the undisturbed vortex ring coincides with the $z$ axis. The numerical domain is $0 \leqslant r \leqslant L_{r}, 0 \leqslant \theta \leqslant 2 \pi$ and $0 \leqslant z \leqslant L_{z}$. Periodic boundary conditions are assumed in the $z$ direction; the spatial period in the $z$ direction is taken sufficiently long so that the growth rate of an unstable mode is nearly the same as that for the infinitely long case.

The task consists of two steps: first, a quasi-steady base flow is obtained by solving the axisymmetric Navier-Stokes equations; next, the linearized Navier-Stokes equations are 
integrated for a sufficiently long time to obtain the most unstable mode when it exists. The details are described below.

\subsection{Numerical methods}

The incompressible Navier-Stokes equations are written as

$$
\begin{aligned}
\frac{\partial \boldsymbol{u}}{\partial t}+\boldsymbol{u} \cdot \nabla \boldsymbol{u} & =-\nabla p+\nu \nabla^{2} \boldsymbol{u}, \\
\boldsymbol{\nabla} \cdot \boldsymbol{u} & =0
\end{aligned}
$$

where $\boldsymbol{u}$ is the velocity, $p$ is the pressure and $\nu$ is the kinematic viscosity; the constant density is set to $\rho_{0}=1$. To obtain a base flow, we set $\boldsymbol{u}=u(t, r, z) \boldsymbol{e}_{r}+v(t, r, z) \boldsymbol{e}_{\theta}+$ $w(t, r, z) \boldsymbol{e}_{z}$, where $\boldsymbol{e}_{r}, \boldsymbol{e}_{\theta}$ and $\boldsymbol{e}_{z}$ are the unit vectors in the $r, \theta$ and $z$ directions; it is pointed out that $v(r, z)$ is included since we consider a vortex ring with swirl in general. The resulting axisymmetric Navier-Stokes equations are solved numerically. For the spatial discretization, the sixth-order accurate compact scheme (Lele 1992) is used in the $r$ direction, while the Fourier spectral method is used in the $z$ direction, in which the periodic boundary conditions are imposed. The singularity at $r=0$ is avoided by expanding the $r$ axis to $-L_{r} \leqslant r \leqslant L_{r}$ and placing no grid point at $r=0$. The Poisson equation can be decomposed into a set of ordinary differential equations for a single Fourier mode, which are also solved by the sixth-order accurate compact scheme. For the temporal discretization, the Crank-Nicolson scheme is used for the viscous terms, while the second-order Adams-Bashforth method is used for the other terms. For the details see Appendix A.

To obtain the unstable mode, we solve the linearized Navier-Stokes equations

$$
\begin{aligned}
\frac{\partial \boldsymbol{u}^{\prime}}{\partial t}+\boldsymbol{u}_{b} \cdot \boldsymbol{\nabla} \boldsymbol{u}^{\prime}+\boldsymbol{u}^{\prime} \cdot \boldsymbol{\nabla} \boldsymbol{u}_{b} & =-\nabla p^{\prime}+\nu \nabla^{2} \boldsymbol{u}^{\prime}, \\
\boldsymbol{\nabla} \cdot \boldsymbol{u}^{\prime} & =0,
\end{aligned}
$$

where $\left(\boldsymbol{u}^{\prime}, p^{\prime}\right)$ is the linear disturbance and $\boldsymbol{u}_{b}$ is the base flow which is obtained numerically and is frozen in time after the relaxation process; this frozen-in-time assumption is valid because the time scale of viscous diffusion is much larger than the characteristic time of instabilities and is justified by DNS in $\S 5$. Since the base flow is axisymmetric, the above equations are separable for Fourier modes in $\theta$. Thus we take $\boldsymbol{u}^{\prime}=\hat{\boldsymbol{u}}(r, z) e^{\mathrm{i} N_{\theta} \theta}$, where $N_{\theta}$ is a positive integer. The same methods of discretization as those for the base flow are employed. In Appendix B, the accuracy of the numerical method is checked.

\subsection{Initial conditions}

To obtain a base flow, we start with Gaussian distributions for both vorticity $\omega_{\theta}$ and swirl velocity $v$. After a transient time evolution the distributions converge to quasisteady state; the details are shown in $\S 3$.

To obtain an unstable mode, the vorticity distribution of the disturbance is randomized initially for $R e=10^{4}$. The disturbance energy decays at the early stage of time evolution as irrelevant modes decay. However, as unstable modes grow the energy tends to an exponential growth. The most unstable eigenmode is obtained approximately after a sufficiently long time $t=100 \sim 200\left(R / u_{\phi \text {, max }}\right)$, where $R$ and $u_{\phi \text {, max }}$ are the radius of the vortex ring and the maximum circumferential velocity, respectively. On the other hand, the initial vorticity distribution for $R e=5 \times 10^{4}$ is set to the unstable mode already obtained for a case close to the case under consideration. In this case the disturbance energy settles quickly to an exponential growth and the computational time is reduced. 


\subsection{Simulation parameters}

The parameters which should be specified in the present problem are summarized here. The base flow is characterized by the circulation $\Gamma$ and the radius $R$ of the vortex ring, the radius $a$ of the vortex core and the maximum swirl velocity $v_{\max }$. The centre of the vortex core is defined as the position $\left(r_{c}, z_{c}\right)$ at which Stokes' streamfunction is maximum in the frame moving with the vortex ring; then the ring radius is given by $R=r_{c}$ and the core radius is defined by $a^{2}=\int\left[\left(r-r_{c}\right)^{2}+\left(z-z_{c}\right)^{2}\right] \omega_{\theta} d r d z / \int \omega_{\theta} d r d z$. Three nondimensional parameters are important: the Reynolds number $R e=\Gamma /(2 \pi \nu)=\Omega_{\max }^{(0)} a_{0}^{2} / \nu$, the thickness or ratio of the core to radius of the $\operatorname{ring} \varepsilon=a / R$ and the swirl parameter $W=0.638 v_{\max } / u_{\phi, \max } \approx v_{\max } /\left(\Omega_{\max }^{(0)} a_{0}\right)$, where the numerical factor 0.638 is peculiar to the Gaussian distribution of vorticity. Here, $a_{0}$ is the initial core radius and $u_{\phi \text {, } m a x}$ and $\Omega_{\max }^{(0)}=\Gamma /\left(2 \pi a_{0}^{2}\right)$ are the maximum circumferential velocity and the maximum angular velocity around the vortex core, respectively; it is pointed out that $\Omega_{\max }^{(0)}$ is defined for the initial Gaussian vorticity distribution as in Blanco-Rodríguez \& Le Dizès (2017).

The parameters listed above change in general as time proceeds and the base flow settles to a quasi-steady state. The circulation is nearly conserved, which implies the Reynolds number is nearly constant. The thickness grows in time, while the swirl parameter decays. How they evolve is shown in $\S 3$ along with how to set parameter values. In $\S 4$ we choose the core radius $a$ as the length scale and the inverse of the angular velocity $1 / \Omega_{\max }^{(0)}$ as the time scale; all quantities are non-dimensionalized using these scales unless stated explicitly.

The disturbance is characterized by the wavenumber $N_{\theta}$ in the $\theta$ direction. The wavenumber is scaled as

$$
k=N_{\theta} a / R=\varepsilon N_{\theta},
$$

when the length of the circle along the centre of the vortex ring $2 \pi R$ is nondimensionalized by $a$. The relation (2.5) implies that the wavenumber $k$ is quantized for fixed $\varepsilon$ since $N_{\theta}$ is an integer. On the other hand, we want to change $k$ continuously in order to explore the curvature and elliptic instability, both of which are due to parametric resonance of two waves. Thus we allow $\varepsilon$ to vary around a prescribed value. For example, the wavenumber $k$ is 1.144 at one of the crossing points of the dispersion curves of $m=0$ and $m=1$ waves giving rise to curvature instability; we choose $N_{\theta}=11$ for a prescribed value of $\varepsilon_{0}=0.1$, which gives the actual value $\varepsilon=k / N_{\theta}=0.104$. The difference from the prescribed value of the thickness is bounded as $\left|\varepsilon-\varepsilon_{0}\right| / \varepsilon_{0} \lesssim 1 /\left(2 N_{\theta}\right)=\varepsilon /(2 k)$ (Gargan-Shingles, Rudman \& Ryan 2016); this difference is sufficiently small after the growth rate is scaled taking account of dependence on $\varepsilon$.

The actual values of the base flow parameters are chosen as listed in Table 1 . In order to study curvature instability, we consider thin vortex rings at high Reynolds numbers. We set the Reynolds number to $R e=10^{4}$, while $R e=5 \times 10^{4}$ is also used for comparison. The prescribed values of the thickness are $\varepsilon_{0}=0.1$ and 0.18 , while $\varepsilon_{0}=0.05$ is also used for a limited number of cases. The swirl parameter is set to $W=0,0.2$ and 0.4 as in Blanco-Rodríguez \& Le Dizès (2017); in this range of $W$ the swirl is sufficiently small that the swirling jet instability is not present. The range of wavenumber is set to $1 \leqslant k \leqslant 4$ or $1 \leqslant k \leqslant 4.5$ depending on the case.

The radial size of the domain is set to $L_{r}=100 R_{0}$, where $R_{0}$ is the initial radius of the vortex ring. Slip boundary conditions are imposed at $r=L_{r}$. Imposing slip boundary conditions is equivalent to placing virtual vortices called image vortices outside the domain (Saffman 1992). The strength and the radial position of the image vortices 


\begin{tabular}{ccc}
\hline \hline$\varepsilon_{0}$ & $R e$ & $W$ \\
\hline 0.1 & $10^{4}$ & 0 \\
& & 0.2 \\
& & 0.4 \\
& $5 \times 10^{4}$ & 0.2 \\
\hline 0.05 & $10^{4}$ & 0.2 \\
0.18 & $10^{4}$ & 0.2 \\
\hline \hline
\end{tabular}

TABLE 1. Base flow parameters.

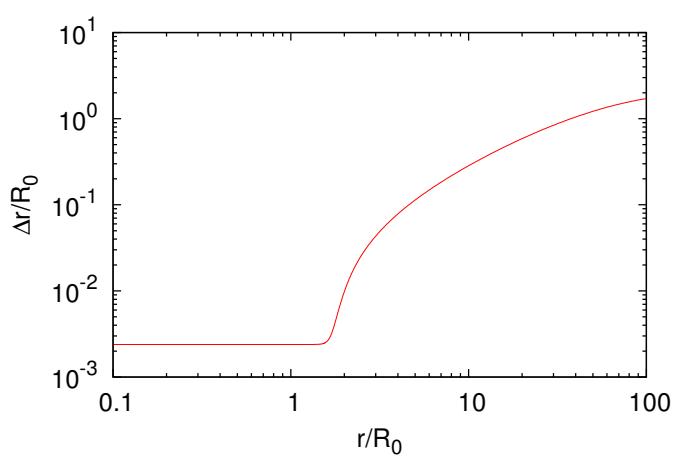

Figure 2. Grid spacing in $r$ direction. Grid B.

become weaker and farther, respectively, as $L_{r}$ increases. Therefore, the outer boundary is set sufficiently far to make the effect of the image vortices negligible; see Appendix B for dependence on $L_{r}$. This large value of $L_{r}$ does not lead to a large number of grid points since non-uniform stretched grids are used in the $r$ direction (figure 2); the grid size at $r=L_{r}$ is $\Delta r=2 R_{0}$. The axial size of the domain is set to $L_{z}=4 \pi / 3 R_{0}$ with periodic boundary conditions. This means that we actually simulate a row of vortex rings. However, for this value of $L_{z}$, the effect of distant rings remains small compared to the self-induced effect of the ring on itself. The grid parameters are listed in Table 2. The minimum grid size $\Delta r_{\text {min }}$ in the $r$ direction and the uniform grid spacing $\Delta z$ in the $z$ direction should be small enough to resolve waves on the vortex core; for $R e=10^{4}$ the minimum number of grid points within the diameter of the vortex core are 83 and 98 in the $r$ and $z$ directions, respectively, while the numbers of points are doubled for $R e=5 \times 10^{4}$ since fine structures may appear as viscous diffusion becomes weak. We have confirmed that the grid resolution is sufficient (see Appendix B). The time step $\Delta t$ is set proportional to the minimum grid size (Table 2); for the dependence on the time step, also see Appendix B, which shows that it is sufficiently small to give accurate results. The total time of integrating the linearized equations is $100 \sim 200\left(R / u_{\phi, \max }\right)$, for which the error of the growth rate of a curvature instability mode is less than $0.01 \%$. 


\begin{tabular}{ccccccc}
\hline \hline Grid type & $\Delta r_{\min } / R_{0}$ & $\Delta z / R_{0}$ & $N_{r}$ & $N_{z}$ & $u_{\phi, \max } \Delta t / R_{0}$ & $\left(\varepsilon_{0}, R e\right)$ \\
\hline Grid A & $5 \times 10^{-3}$ & $4.09 \times 10^{-3}$ & 694 & 1024 & $5 \times 10^{-4}$ & $\left(0.18,10^{4}\right)$ \\
Grid B & $2.5 \times 10^{-3}$ & $2.05 \times 10^{-3}$ & 946 & 2048 & $2.5 \times 10^{-4}$ & $\left(0.1,10^{4}\right)$ \\
Grid C & $1.25 \times 10^{-3}$ & $1.02 \times 10^{-3}$ & 1346 & 4096 & $1.25 \times 10^{-4}$ & $\left(0.05,10^{4}\right),\left(0.1,5 \times 10^{4}\right)$ \\
\hline \hline
\end{tabular}

TABLE 2. Grid parameters.
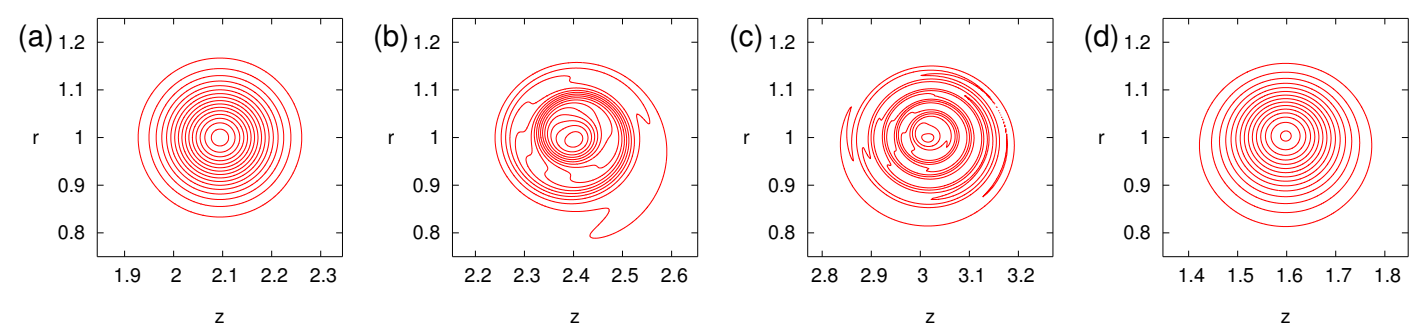

Figure 3. Time evolution of a vortex ring. Contours of $v$. Re $=10^{4}, \varepsilon=0.1, W=0.2$. $t=0,1,3,12$. Contour levels are $v / W=0.0638 n(n=1,2, \cdots)$.

\section{Base flow}

In this section we show how we obtain the quasi-steady state of the vortex ring that is used as a base flow. Starting from Gaussian distributions of azimuthal vorticity and swirl velocity (figure 3), the vortex ring reaches a quasi-steady state after a certain time of evolution. There are subtle issues on controling parameters $\varepsilon$ and $W$ since they both evolve in time; how we control them is also explained.

Figure 3 shows the time evolution of a vortex ring by contours of $v$ for $R e=10^{4}, W=$ 0.2 . After transient evolution (figure $3 \mathrm{~b}, \mathrm{c}$ ) the vortex ring reaches a quasi-steady state by $t=12$ (figure 3d). For an exact steady state of inviscid axisymmetric flow $\omega_{\theta} / r$ becomes a function of Stokes' streamfunction $\Psi$ when $W=0$, while $r v$ becomes a function of $\Psi$ when $W \neq 0$. This is confirmed in figure 4 which shows scatter plots of $\Psi$ and $r v$. The thickness of the scatter plot becomes small as time proceeds; at $t=12$ we obtain a good functional relation between $\Psi$ and $r v$. We also confirmed a functional relation between $\Psi$ and $\omega_{\theta} / r$ for $W=0$, but this result is not shown here since it is well documented in previous works (Shariff, Verzicco \& Orlandi 1994). Quasi-steadiness is also checked by the $L^{2}$-norm of

$$
\Delta \omega_{\theta}=\omega_{\theta}(t+\Delta t, r, z+U \Delta t)-\omega_{\theta}(t, r, z),
$$

which is the difference of the azimuthal vorticity between two adjacent time steps (figure 5 ); here $U$ is the translating velocity (figure $6 \mathrm{~b}$ ). It is observed that the difference decreases as time proceeds and becomes close to zero after $t \approx 10$.

Figure 6 shows the time evolution of squared core radius and translating velocity of a vortex ring. The vortex core spreads due to viscous diffusion as

$$
a^{2}(t)=a_{0}^{2}+4 \nu t
$$

after a transient behaviour which depends on the initial conditions (initial transient). The 

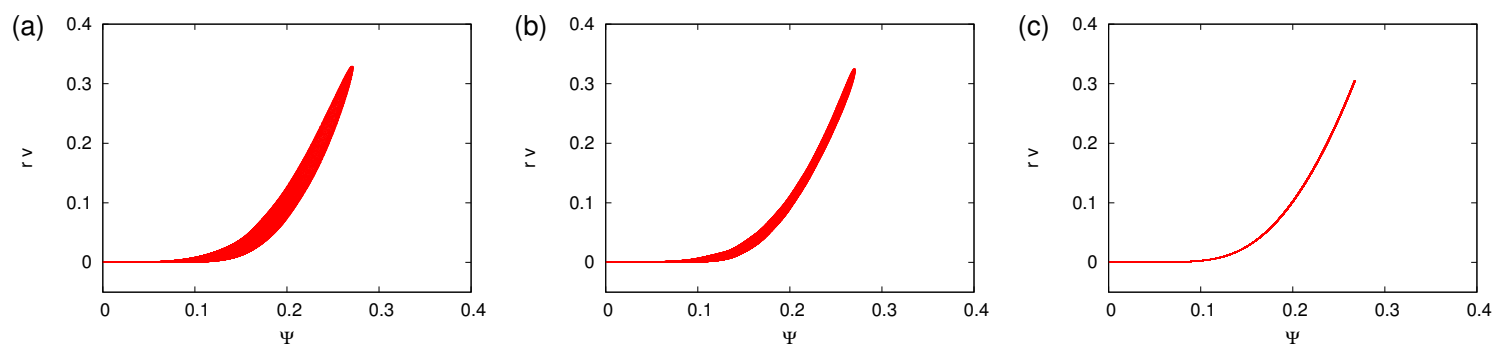

Figure 4. Scatter plot of $\Psi$ and $r v . R e=10^{4}, \varepsilon=0.1, W=0.2 . t=1,3,12$.

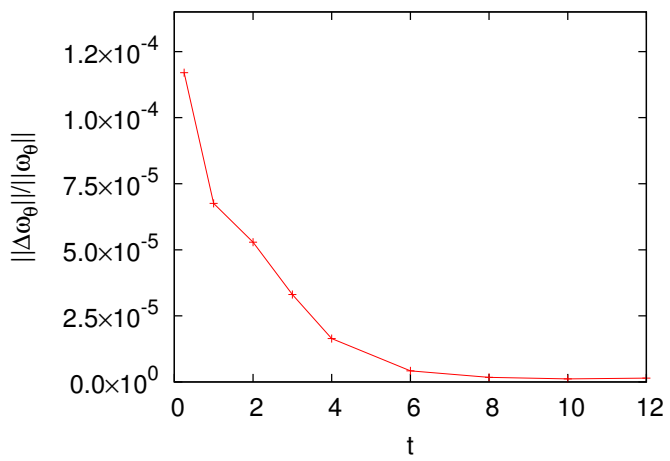

FiguRe 5. $L^{2}$-norm of $\Delta \omega_{\theta}=\omega_{\theta}(t+\Delta t, r, z+U \Delta t)-\omega_{\theta}(t, r, z) . R e=10^{4}, \varepsilon=0.1, W=0.2$.

(a)

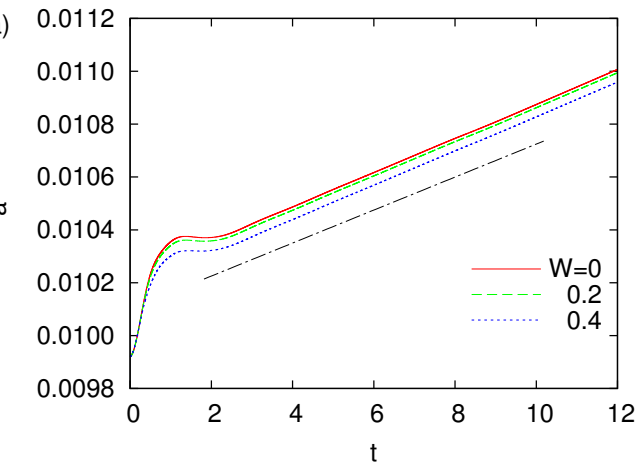

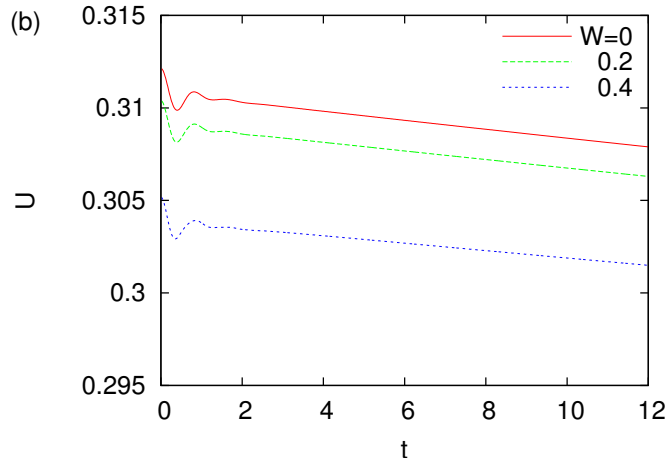

Figure 6 . Time evolution of (a) squared core radius and (b) translating velocity. $R e=10^{4}, \varepsilon=0.1, W=0,0.2,0.4$. The slope of the dot-dashed line in figure $6(\mathrm{a})$ is $4 \nu$.

increase of the core radius affects resonance condition of curvature and elliptic instabilities since the wavenumber non-dimensionalized by the core radius determines the condition. This figure also shows the effects of swirl: both the core radius and translating velocity decrease slightly for larger swirl. The latter result is in agreement with analytical results (Widnall Bliss \& Zalay 1971).

Figure 7 shows time evolution of the swirl parameter $W=0.638 v_{\max } / u_{\phi, \max }$. The swirl velocity decreases monotonically after an initial transient. This result is explained as follows. Since the angular momentum $A_{z} \approx v_{\max } \pi a^{2} R$ is approximately constant, we 
(a)

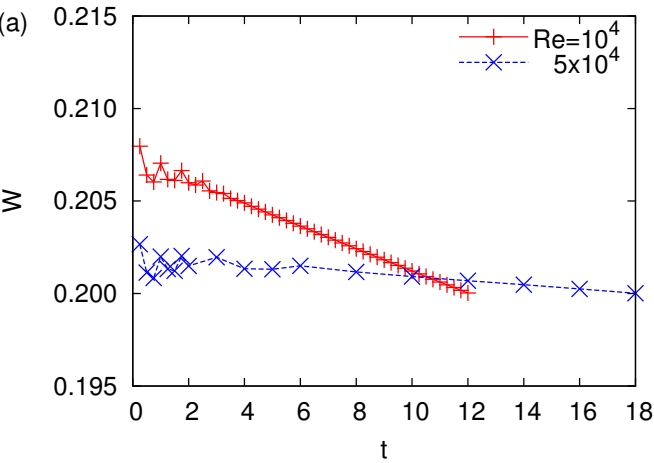

(b)

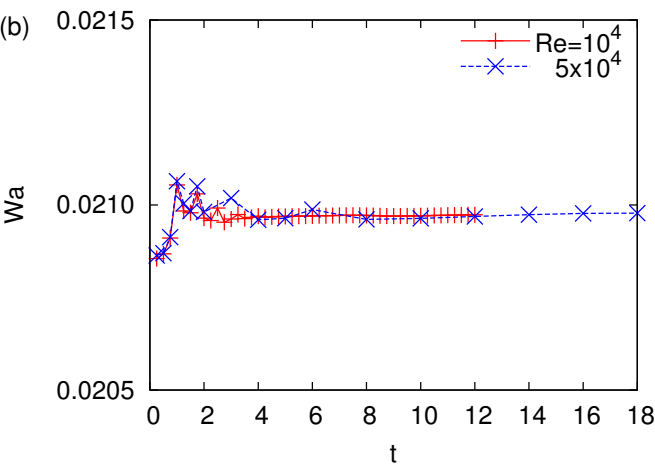

Figure 7. Time evolution of swirl parameter. $R e=10^{4}, 5 \times 10^{4}, W \approx 0.2$. (a) $W$ and (b) $W a$.

have

$$
v_{\max } \propto \frac{1}{a^{2}}
$$

when the ring radius is nearly constant. On the other hand, since the circulation is also approximately constant, we have

$$
u_{\phi, \max } \propto \frac{\Gamma}{2 \pi a} .
$$

Therefore, the ratio decreases with time as $W \propto a^{-1}$, which is confirmed in figure $7(\mathrm{~b})$. The initial values of the core radius and the swirl parameter are determined taking account of their time evolutions so that they become prescribed values when the flow becomes quasi-steady. The results shown below are based on the quasi-steady states at $t=12$ and 18 for $R e=10^{4}$ and $5 \times 10^{4}$, respectively.

\section{Results}

In this section we show linear stability results. First, the overall features of the linear instability are shown by growth rate and oscillation frequency plotted against azimuthal wavenumber; mode structures are identified for some typical unstable modes. Next, we focus on two curvature instability modes to make comparison between numerical and theoretical results. Finally, other modes of interest are shown and discussed.

\subsection{Growth rate}

The unstable modes obtained at large $t$ are proportional to $e^{(\sigma+\mathrm{i} \omega) t}$. The growth rate $\sigma$ and oscillation frequency $\omega$ are shown as a function of the azimuthal wavenumber $k$ in figures $8-11$. The Reynolds number is fixed at $10^{4}$. The thickness $\varepsilon$ is approximately 0.1 in figures $8-10$ and 0.18 in figure 11. The swirl parameter $W$ is $0,0.2$ and 0.4 in figures 8,9 and 10, respectively, while it is also set to 0.2 in figure 11 .

Several peaks of the growth rate are observed in each case; the width of the peaks is narrow for $\varepsilon_{0}=0.1$ as the instability is mostly due to parametric resonance; it becomes wide for $\varepsilon_{0}=0.18$ as the width is essentially proportional to $\varepsilon_{0}$. In these figures typical modes are identified by their structures. The instability is due to either the curvature instability (marked by solid circles and crosses) or the elliptic instability (marked by open circles) except for the spiral modes (marked by diamonds for $W=0.4$ ). Most of the modes are composed of two inertial waves which are indexed by the branch of 
(a)

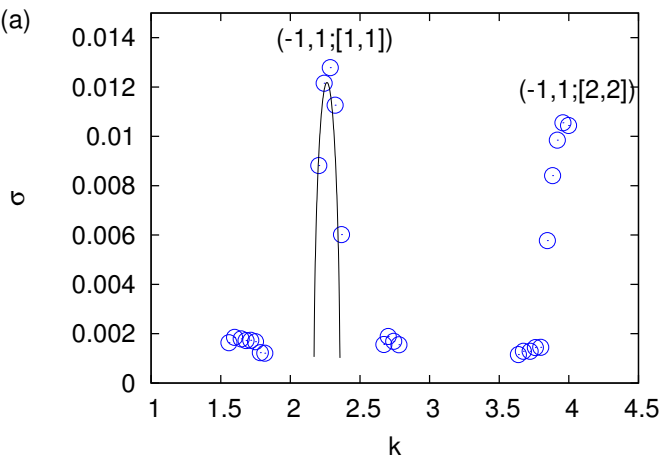

(b)

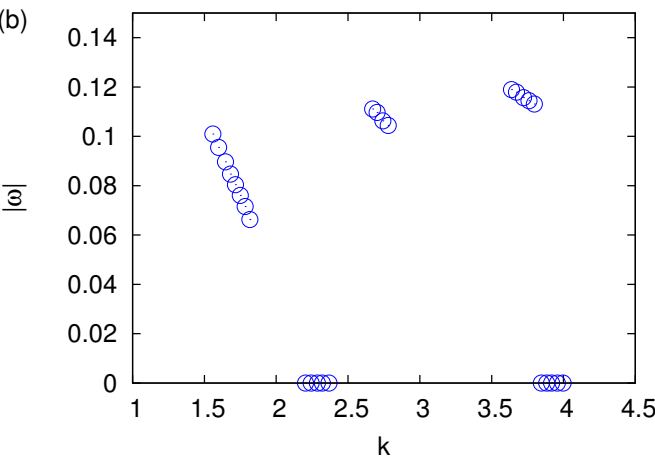

FiguRe 8. (a) Growth rate $(\sigma=\operatorname{Im}(\omega))$ and (b) frequency $(\operatorname{Re}(\omega))$ plotted against azimuthal wavenumber. $\varepsilon_{0}=0.1$. $R e=10^{4}, W=0$. All modes are due to elliptic instability marked by open circles. The lines show theoretical values (Blanco-Rodríguez \& Le Dizès 2016).

(a)

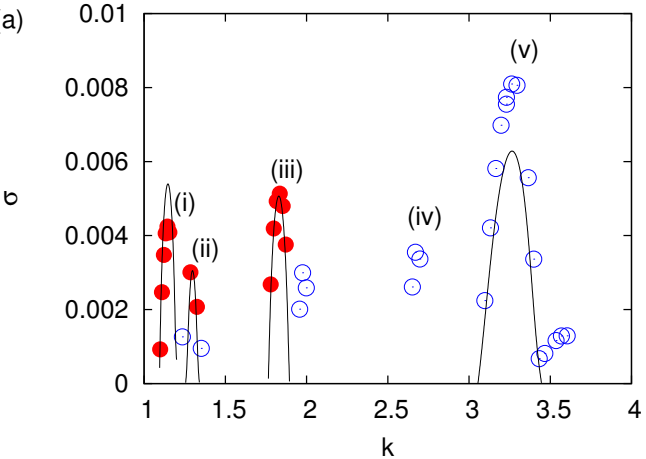

(b)

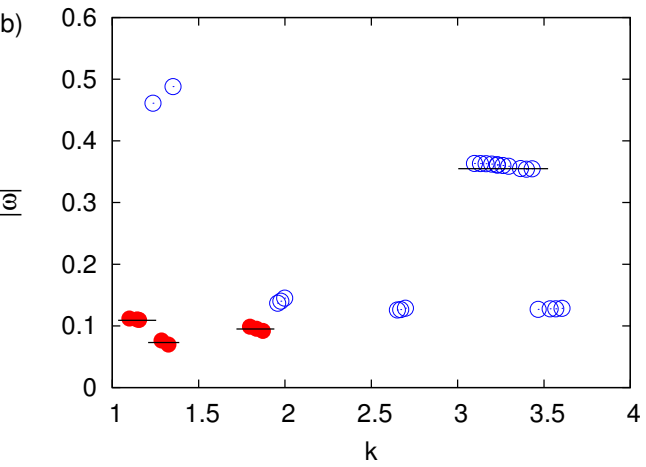

Figure 9. (a) Growth rate and (b) frequency plotted against azimuthal wavenumber. $\varepsilon_{0}=0.1$. $R e=10^{4}, W=0.2$. Solid circles: curvature instability, open circles: elliptic instability. The lines show theoretical values (Blanco-Rodríguez \& Le Dizès 2016, 2017). Some typical modes are identified: (i) $(-1,0 ;[1,3])$, (ii) $(-1,0 ;[1,4])$, (iii) $(-1,0 ;[2,4])$, (iv) $(-1,1 ;[2,1])$, (v) $(-2,0 ;[2,2])$.

dispersion curves. For example, the most unstable mode for $\varepsilon_{0}=0.1$ and $W=0$ (figure 8 ) is due to elliptic instability and consists of the $m= \pm 1$ inertial waves of the first branches which crosses at $(k, \omega)=(2.333,0)$ so that it is labeled as $(-1,1 ;[1,1])$. The mode labeled by (i) for $\varepsilon_{0}=0.1$ and $W=0.2$ (figure 9 ) is due to curvature instability and consists of the $m=-1$ inertial wave of the first branch and the $m=0$ inertial wave of the third branch, which crosses at $(k, \omega)=(1.14,-0.11)$, so that it is denoted as $(-1,0 ;[1,3])$. It is pointed out that the sign of $m$ and thereby the order of the indices of the branches are different from Blanco-Rodríguez \& Le Dizès (2017) since we choose $k$ to be positive in the present work: $(-1,0 ;[1,3])$ with $(k, \omega)=(1.14,-0.11)$ in the present work corresponds to $\left(m_{A}, m_{B}\right)=(0,1)$ and $\left(l_{A}, l_{B}\right)=(3,1)$ with $(k, \omega)=(-1.14,0.11)$ in Blanco-Rodríguez \& Le Dizès (2017). Theoretical values for some typical modes taken from Blanco-Rodríguez \& Le Dizès (2016) and Blanco-Rodríguez \& Le Dizès (2017) are also included by lines in the figures $8-11$.

For $\varepsilon_{0}=0.1$ and $W=0$ (figure 8 ) the instability is due to elliptic instability. The growth rate of curvature instability for $W=0$ is too small to be the most unstable mode or be captured by numerical simulation. Two modes have large growth rates; the indices of the branches of the two waves are same and they are often called principal modes. The 
(a)

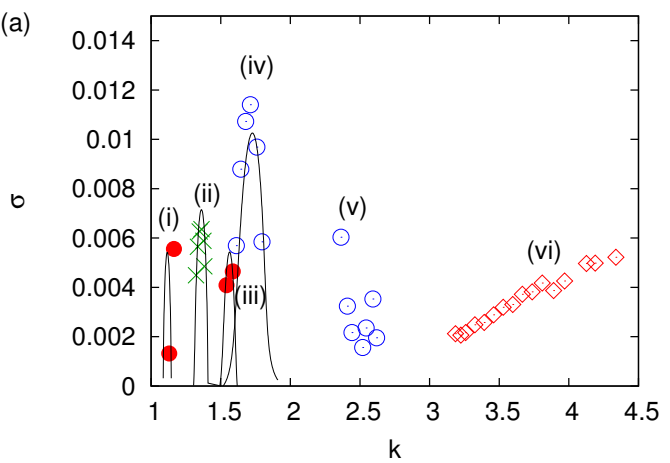

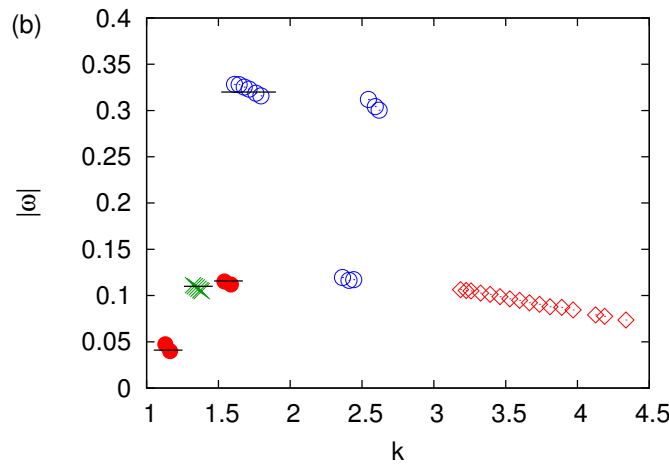

Figure 10. (a) Growth rate and (b) frequency plotted against azimuthal wavenumber. $\varepsilon_{0}=0.1$. $R e=10^{4}, W=0.4$. The symbols and lines are the same as in figure 9 , while the crosses and the diamonds correspond to three-wave modes and spiral modes, respectively. (i) $(-1,0 ;[1,3])$, (ii) three-wave mode, (iii) $(-1,0 ;[3,2])$, (iv) $(-2,0 ;[1,1])$, (v) $(-2,0 ;[1,2])$, (vi) spiral mode.

(a)

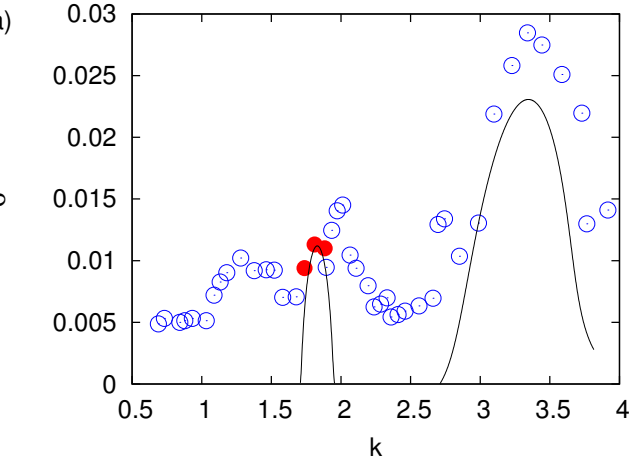

(b)

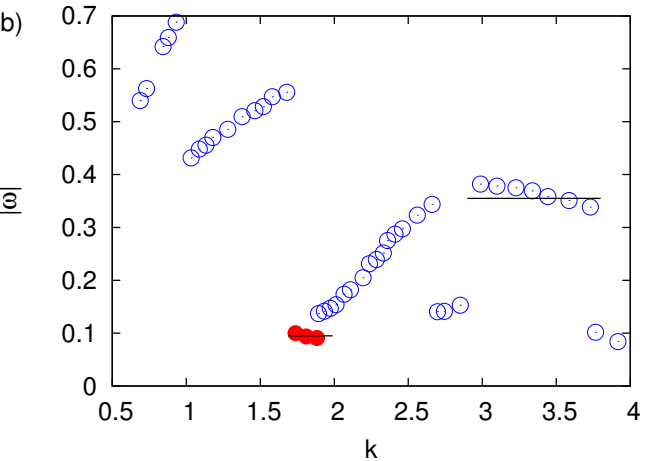

Figure 11. (a) Growth rate and (b) frequency plotted against azimuthal wavenumber. $\varepsilon_{0}=0.18$. $R e=10^{4}, W=0.2$. The symbols and lines are the same as in figure 9 .

frequency is zero for these modes. The values of the growth rate are in good agreement with theory. There are also modes with small growth rate and non-zero frequency; the indices of the two inertial waves are different for these modes.

For $\varepsilon_{0}=0.1$ and $W=0.2$ (figure 9 ) three modes of curvature instability are identified: (i) $(-1,0 ;[1,3])$, (ii) $(-1,0 ;[1,4])$ and (iii) $(-1,0 ;[2,4])$. The other modes are due to elliptic instability; two modes are identified: (iv) $(-1,1 ;[2,1])$ and $(\mathrm{v})(-2,0 ;[2,2])$. They are also found in previous works for a strained vortex and a vortex pair (Lacaze, Ryan \& Le Dizès 2007; Roy, Schaeffer, Le Dizès \& Thompson 2008). It is known that asymmetric modes like $(-2,0 ;[2,2])$ have large growth rate when the swirl is added. In the present range of wavenumber $1 \leqslant k \leqslant 4$, the most unstable mode is the elliptic instability of $(-2,0 ;[2,2])$, while the second most unstable mode is the curvature instability of $(-1,0 ;[2,4])$. All modes are oscillatory having non-zero frequencies. The values of the growth rate are in good agreement for the curvature instability of $(-1,0 ;[2,4])$, while some differences are observed for the elliptic instability of $(-2,0 ;[2,2])$ and the curvature instability of $(-1,0 ;[1,3])$. The growth rate of the curvature instability modes will be compared carefully to theory in the next subsection.

For $\varepsilon_{0}=0.1$ and $W=0.4$ (figure 10) three modes of curvature instability are identified: (i) $(-1,0 ;[1,3])$, (ii) three-wave mode and (iii) $(-1,0 ;[3,2])$. The structure 

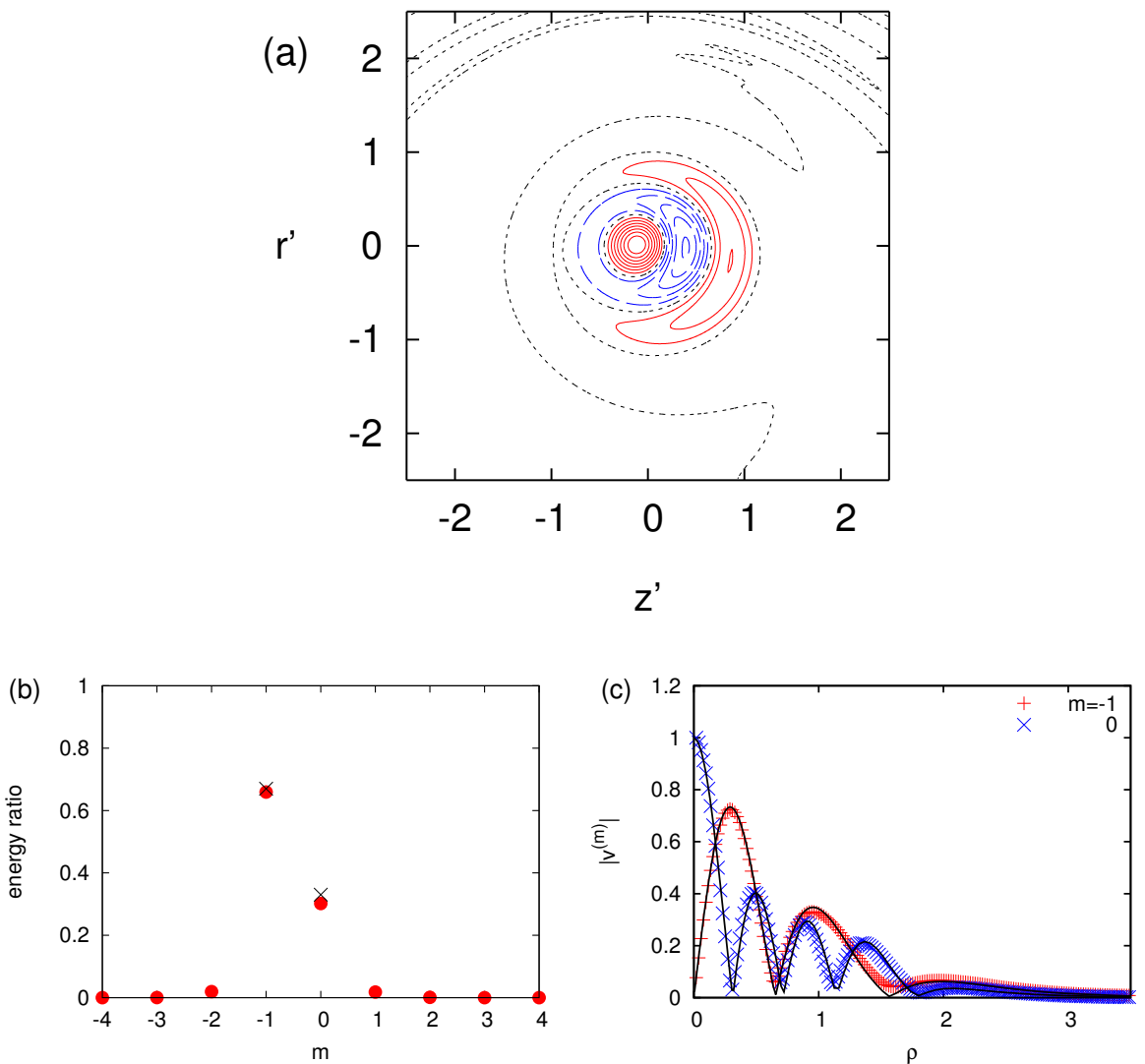

Figure 12. Mode structure. $(-1,0 ;[2,4])$ mode with $(k, \omega)=(1.853,-0.095) . W=0.2$, $R e=10^{4}, \varepsilon_{0}=0.1$. (a) Contours of azimuthal component of vorticity $\omega_{\theta}: \omega_{\theta}>0$ (solid), $=0$ (dotted) and < 0 (dashed). (b) Ratio of energy; solid circles: DNS, crosses: theory. (c) Radial distribution of $v^{(m)}$; symbols: DNS, lines: theory.

of the three-wave mode is detailed later in $§ 4.3 .2$. Three modes of elliptic instability are also observed. Two modes having larger growth rate are identified: (iv) $(-2,0 ;[1,1])$ and (v) $(-2,0 ;[2,1])$ (see also figure 22b). Again these modes are also found in previous works (Lacaze, Ryan \& Le Dizès 2007; Roy, Schaeffer, Le Dizès \& Thompson 2008). The spiral mode is different from the other modes as its growth rate does not have a clear peak; it extends in the wavenumber range $3.2 \leqslant k$. The structure of this mode is discussed in $\S 4.3 .3$.

We show the case $\varepsilon_{0}=0.18$ and $W=0.2$ in figure 11 to see the effects of the core size by comparing to figure 9 . Only one mode of curvature instability $(-1,0 ;[2,4])$ survives as the growth rate of elliptic instability increases as $\varepsilon^{2}$ which is faster than that of curvature instability $\varepsilon$. The band width of each mode broadens as is observed for the most unstable mode at $k \approx 3.4$ due to elliptic instability. As a result the vortex ring is unstable at any wavenumber $k$ in the present range.

\subsection{Typical curvature instability modes and critical layer damping}

Next, we focus on the two modes of curvature instability for $W=0.2:(-1,0 ;[1,3])$ and $(-1,0 ;[2,4])$. Figure 12 shows the structure of the curvature instability mode $(-1,0 ;[2,4])$. Contours of azimuthal vorticity $\omega_{\theta}$ are shown in figure $12(\mathrm{a})$. This figure 


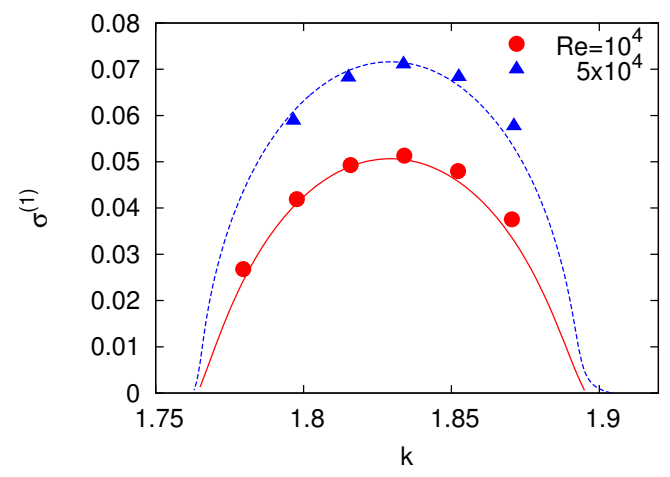

Figure 13. Growth rates. $W=0.2,(-1,0 ;[2,4])$ mode, $\varepsilon_{0}=0.1 . R e=10^{4}, 5 \times 10^{4}$. Symbols: DNS, lines: theory.

is similar to figure 7(e) in Blanco-Rodríguez \& Le Dizès (2017). In order to reveal the mode structure, we decompose the unstable mode as

$$
\hat{\boldsymbol{u}}(r, z)=\sum_{m} \boldsymbol{u}^{(m)}(\rho) e^{\mathrm{i} m \phi},
$$

where $(\rho, \phi)$ is the local polar coordinate system of which origin is the centre of the vortex core of the base flow in the $r z$-plane (figure 1). In figure 12(b) the ratio of energy is plotted against $m$. It shows that this mode consists of $m=-1$ and $m=0$ waves and the values are in good agreement with theory. The structure of each wave is shown by the radial distribution of the azimuthal component of the velocity field $v$ in figure $12(\mathrm{c})$. The number of points where the magnitude of the wave vanishes $(r=0$ excluded) coincides with the index of the branch; in the present case the $m=-1$ wave has two zeros, while the $m=0$ wave has four zeros. Thus the mode is labelled as $(-1,0 ;[2,4])$. Theoretical results are also shown in figure 12(c); they are in excellent agreement with the numerical results.

Figure 13 compares the growth rate with theory (Blanco-Rodríguez \& Le Dizès 2017) for the curvature instability mode $(-1,0 ;[2,4])$. In this figure the growth rate is divided by $\varepsilon$ : $\sigma^{(1)}=\sigma / \varepsilon$. They are also in good agreement having approximately same maximum and band width. It is pointed out that the critical layer damping is small for this mode (Blanco-Rodríguez \& Le Dizès 2017).

Figure 14 shows the structure of the curvature instability mode $(-1,0 ;[1,3])$. Again the vorticity distribution is similar to the theoretical results (Blanco-Rodríguez \& Le Dizès 2017). This mode consists of the $m=-1$ inertial wave of the first branch and the $m=0$ inertial wave of the third branch (figure 14b,c). The radial distributions are in good agreement between the theoretical and numerical results (figure 14c).

Figure 15(a) compares the growth rate with theory (Blanco-Rodríguez \& Le Dizès $2017)$ for the curvature instability mode $(-1,0 ;[1,3])$. In contrast to figure 13 for the mode $(-1,0 ;[2,4])$, the numerical results are smaller than the theoretical values for $\varepsilon_{0}=$ 0.1 ; the maximum growth rate is $\sigma^{(1)}=0.0424$, which is $22 \%$ smaller than the theoretical value $\sigma^{(1)}=0.0541$; it is worth noting that the difference between the numerical and theoretical values does not depend on the Reynolds number very much: for $R e=5 \times 10^{4}$, the maximum growth rate is $\sigma^{(1)}=0.0549$ and the theoretical value is $\sigma^{(1)}=0.0646$; the difference 0.0097 for $R e=5 \times 10^{4}$ is close to 0.0117 for $R e=10^{4}$. It suggests that the difference is not due to viscous diffusion. However, the difference becomes small for 

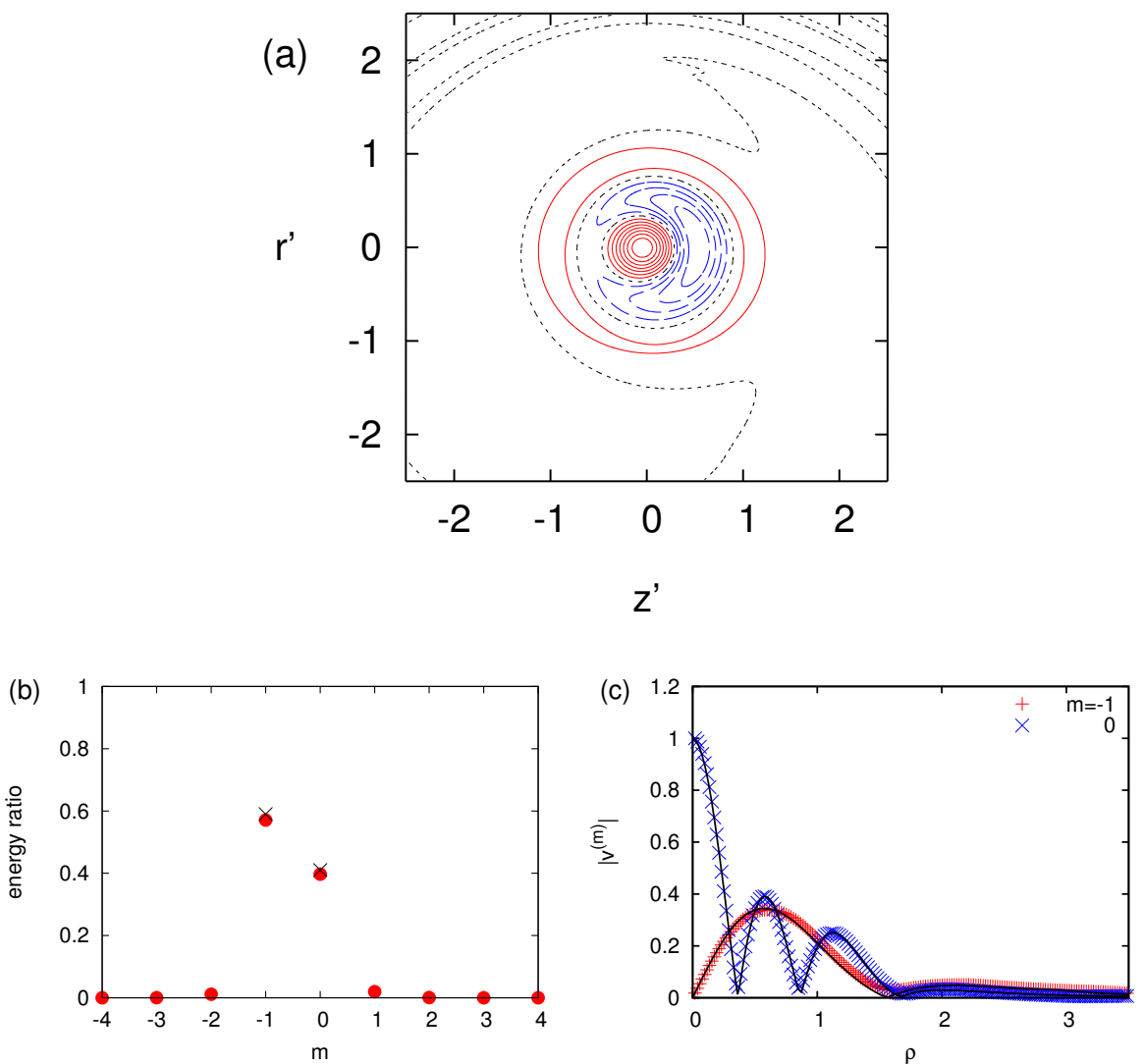

Figure 14. Mode structure. $(-1,0 ;[1,3])$ mode with $(k, \omega)=(1.144,-0.110) . W=0.2$, $R e=10^{4}, \varepsilon_{0}=0.1$. (a) Contours of azimuthal component of vorticity $\omega_{\theta}: \omega_{\theta}>0$ (solid), $=0$ (dotted) and $<0$ (dashed). (b) Ratio of energy; solid circles: DNS, crosses: theory. (c) Radial distribution of $v^{(m)}$; symbols: DNS, lines: theory.
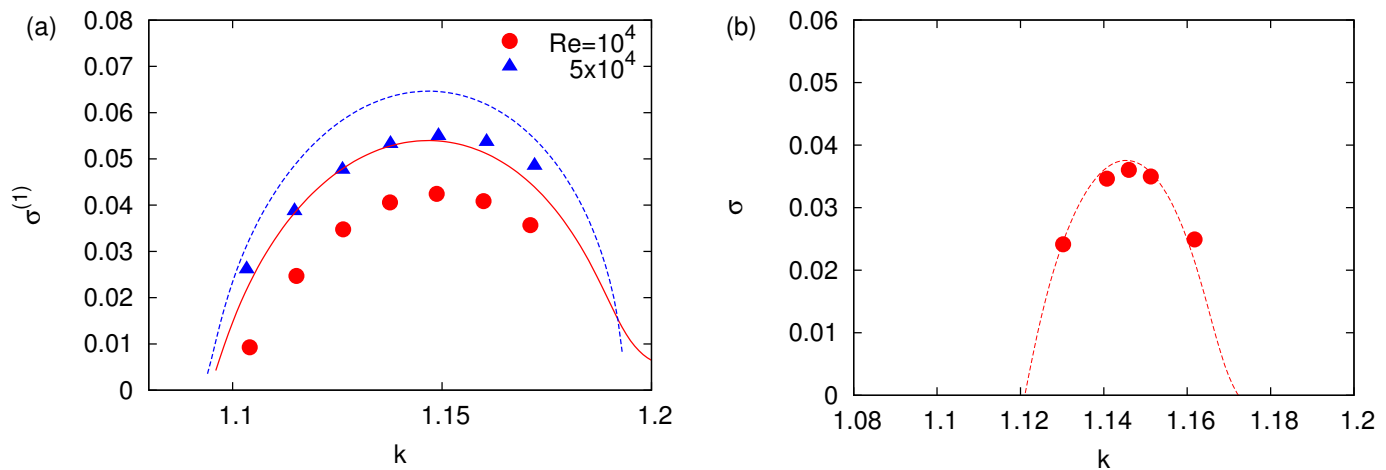

FiguRE 15. Growth rates. $W=0.2,(-1,0 ;[1,3])$ mode. (a) $\varepsilon_{0}=0.1, R e=10^{4}, 5 \times 10^{4}$, (b) $\varepsilon_{0}=0.05, R e=10^{4}$. Symbols: DNS, lines: theory. 
(a)

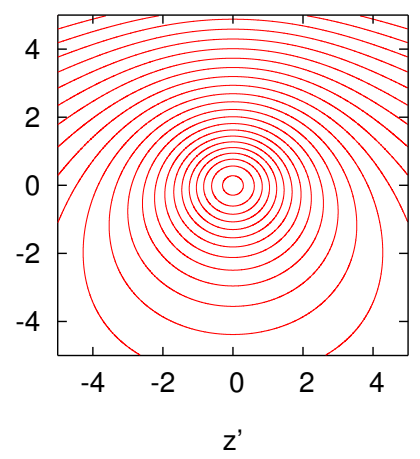

(b)

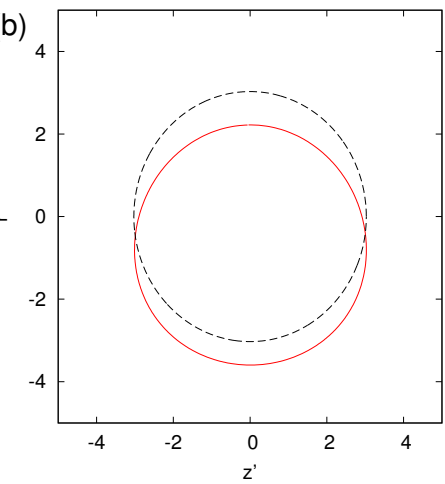

(c)

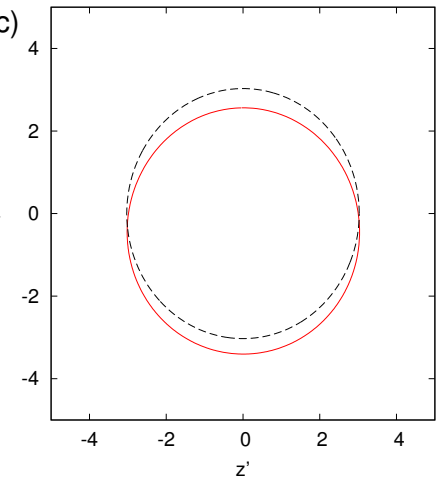

Figure 16. (a) Streamlines of the base flow and (b,c) approximated (solid line) and theoretical (dashed line) positions of the critical layers of the inertial wave of $m=-1$ and $(k, \omega)=(1.14,-0.11), R e=10^{4}, W=0.2$. (a,b) $\varepsilon_{0}=0.1, t=12,(\mathrm{c}) \varepsilon_{0}=0.05, t=16$.

small core $\varepsilon_{0}=0.05$ (figure 15b). It is most likely that the difference for $\varepsilon_{0}=0.1$ is due to the change in the critical layer damping as discussed below.

Theoretically, the critical layer damping is evaluated for the inertial waves on a straight axisymmetric vortex. However, the core of a vortex ring is deformed because of the curvature effects (figures 3 and 16a); deformation is significant for larger $\varepsilon$. We resort to a heuristic argument to estimate the effect of deformation on the critical layer damping since rigorous analysis is difficult and beyond the scope of the present paper. It would be reasonable to approximate the critical layer on a deformed core by a streamline on which the averaged angular velocity $\bar{\Omega}$ of a fluid particle satisfies an averaged version of equation (1.1)

$$
\omega-k \overline{u_{\phi}}-m \bar{\Omega}=0 .
$$

Figures 16(b) and 16(c) compare the approximated and theoretical critical layers for the $m=-1$ inertial wave of $(k, \omega)=(1.14,-0.11)$. The approximated critical layers are subjected to three types of deformation: they change in size (radius), shift downward and are elliptically deformed. Table 3 quantifies the deformation, where the elliptical deformation is measured by the aspect ratio which is the ratio of the short to long axis. On the other hand, the critical layer damping depends crucially on the position of the critical layer. Figure 17 shows the critical layer damping, which is the imaginary part of $\omega$, plotted against the wavenumber $k$ for the first and second branches of the $m=-1$ inertial wave. The modes corresponding to the crossing points $(-1,0 ;[1,3])$ and $(-1,0 ;[2,4])$ are marked by symbols. A simple calculation shows that the theoretical growth rate almost agrees with the numerical value for $(-1,0 ;[1,3])$ if we replace the theoretical critical layer damping -0.00075 by -0.003 . This corresponds to $k=1.07$ in figure 17 , which is $6.1 \%$ smaller than $k=1.14$ at the crossing point. The deformation of the critical layer for $\varepsilon_{0}=0.1$ is $2.0 \%, 23 \%$ and $3.7 \%$ in radius, shift and elliptical deformation, respectively. Therefore, the deformation of the critical layer can change the critical layer damping with the same order of magnitude observed in figure 15(a). Moreover, the change should be small for $\varepsilon_{0}=0.05$ since deformation is small and it should be also small for $(-1,0 ;[2,4])$ since the critical layer damping for the mode $(-1,0 ;[2,4])$ is much smaller than that for $(-1,0 ;[1,3])$ as shown in figure 17 . This heuristic argument explains why the difference between numerical and theoretical growth rates is large in figure 15(a), but not in figures 13 and 15(b), although it should be supported by more rigorous analysis. 


\begin{tabular}{cccc}
\hline \hline & radius & shift & aspect ratio \\
\hline$\varepsilon_{0}=0.1$ & 2.967 & 0.688 & 0.963 \\
$\varepsilon_{0}=0.05$ & 3.005 & 0.420 & 0.985 \\
theory & 3.027 & 0 & 1 \\
\hline \hline
\end{tabular}

TABle 3. Deformation of critical layers.

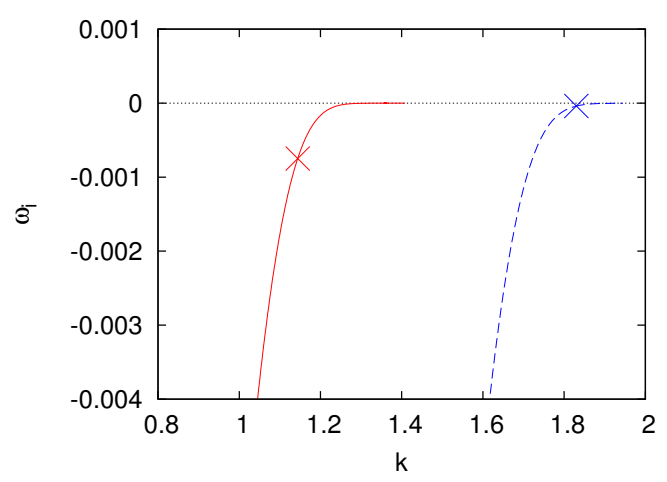

Figure 17. Critical layer damping of the first and second branches. $W=0.2, m=-1$. Solid line: first branch, dashed line: second branch. The crosses correspond to the crossing points $(-1,0 ;[1,3])(k=1.14$ on the first branch) and $(-1,0 ;[2,4])(k=1.83$ on the second branch).

\subsection{Other modes of interest}

Here we focus on some other modes which are typical or interesting by showing the mode structures. The Reynolds number and the thickness are fixed to $10^{4}$ and 0.1 , respectively.

\subsubsection{Elliptic instability}

Figure 18 shows the elliptic instability mode $(-1,1 ;[1,1])$ for $W=0$. It is a well-known mode showing bending deformation in the direction of stretching due to the straining component of the base flow. The two inertial waves are symmetric: the energy ratio of each wave is 0.5 (figure 18b) and the radial distributions of the waves coincide (figure 18c). The radial distributions are in excellent agreement between the theoretical and numerical results.

Figure 19 shows the elliptic instability mode $(-2,0 ;[1,2])$ for $W=0.4$. The $m=-2$ wave of this mode has peaks near $\rho \approx a$, where the radial distribution of this wave is disturbed; differences between the numerical and theoretical results are observed near this point; it may be due to $O\left(\varepsilon^{2}\right)$ effects although it remains to be accounted for. Corresponding narrow peaks are observed near $\rho=a$ in the mode structure (figure 19a).

\subsubsection{Curvature instability}

Figure 20 shows the curvature instability mode $(-1,0 ;[3,2])$ for $W=0.4$. The energy ratio of the $m=0$ wave is larger than that of the $m=-1$ wave; this is contrasted to the two modes of $W=0.2$. Theoretical and numerical results are in good agreement in figure 20(c), confirming that the theory is correct also for $W=0.4$. 

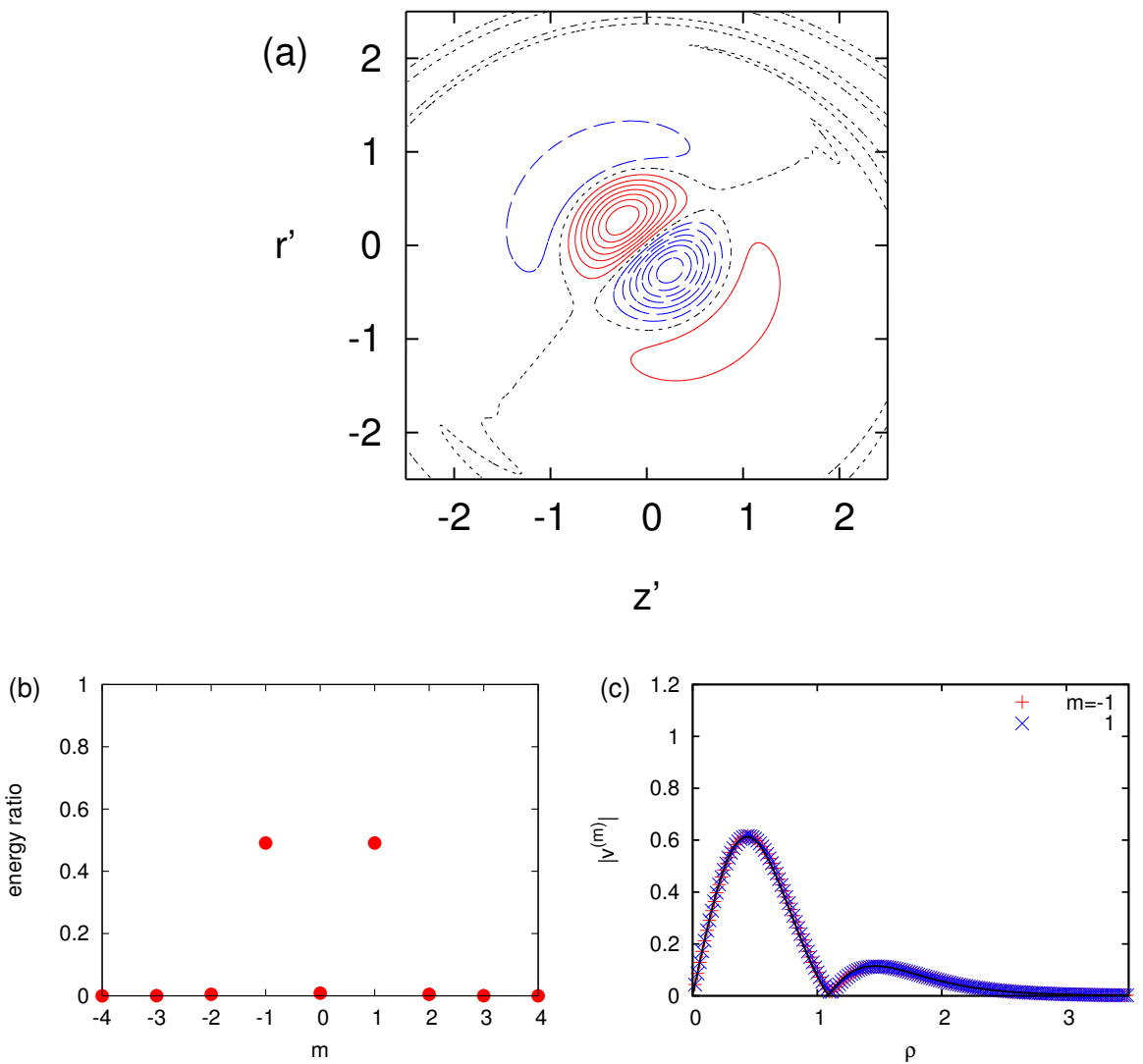

Figure 18. Mode $(-1,1 ;[1,1]), k=0$ (elliptic instability). $W=0, R e=10^{4}, \varepsilon_{0}=0.1$. (a) Contours of azimuthal component of vorticity $\omega_{\theta}: \omega_{\theta}>0$ (solid), $=0$ (dotted) and $<0$ (dashed). (b) Ratio of energy. (c) Radial distribution of $v^{(m)}$; symbols: DNS, lines: theory.

Figure 21 shows the three-wave mode of $(k, \omega)=(1.389,-0.106)$ for $W=0.4$. It consists of the $m=-1,0$ and 1 inertial waves. The energy ratio of the three waves are similar. The displacement mode (Fabre, Sipp \& Jacquin 2006) of the $m=1$ wave happens to come close to the crossing point of the $m=-1$ and $m=0$ waves as shown in figure 22 so that the three waves interact with each other; thus, it is essentially a curvature instability mode. The interaction with the displacement mode would affect the growth rate; although it is of some interest to evaluate it analytically, it is avoided in the present paper since it requires elaborate calculations.

\subsubsection{Spiral mode}

Figure 23 shows the spiral mode with $k=4.231$ for $W=0.4$. It consists of $m=-1$ and $m=0$ waves; the energy ratio of the $m=0$ wave is much larger than that of the $m=-1$ wave. This $m=-1$ wave cannot be identified in the dispersion curves. Ring structures of strong azimuthal vorticity are observed for $1.4 a \lesssim \rho \lesssim 2 a$; correspondingly in this region of $\rho$ the radial distribution of the $m=0$ wave differs between theoretical and numerical results. This spiral mode is similar to those observed in Roy, Schaeffer, Le Dizès \& Thompson (2008). It seems that this kind of modes appears at large wavenumbers and high Reynolds numbers. 

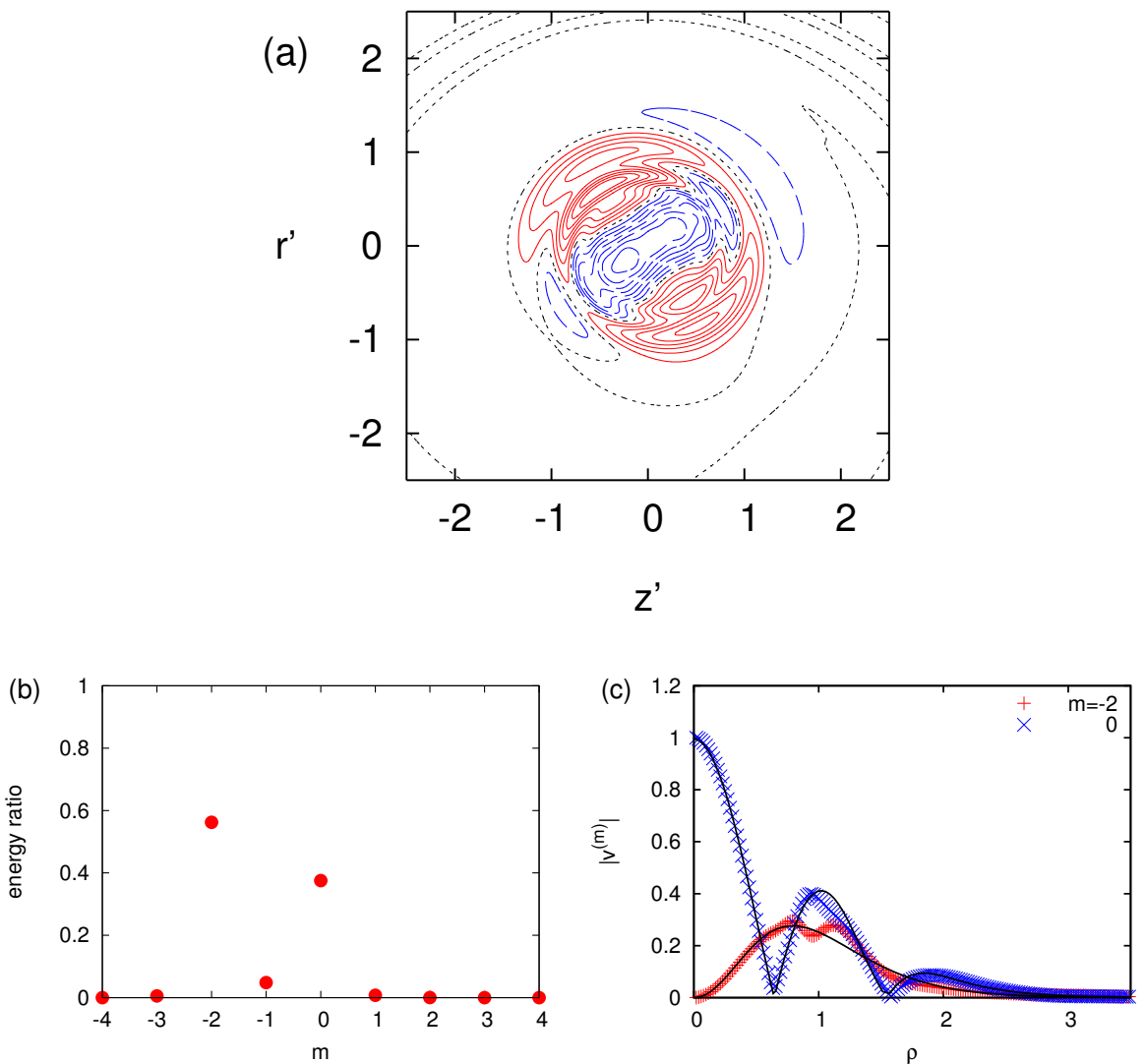

Figure 19. Mode $(-2,0 ;[1,2]), k=2.411, \omega=-0.119$ (elliptic instability). $W=0.4, R e=10^{4}, \varepsilon_{0}=0.1$. (a) Contours of azimuthal component of vorticity $\omega_{\theta}$ : $\omega_{\theta}>0$ (solid), $=0$ (dotted) and $<0$ (dashed). (b) Ratio of energy. (c) Radial distribution of $v^{(m)}$; symbols: DNS, lines: theory.

In order to explore the nature of this instability mode the case with thinner $\left(\varepsilon_{0}=0.05\right)$ and thicker core $\left(\varepsilon_{0}=0.18\right)$ is investigated. For $\varepsilon_{0}=0.05$, no instability is found. For $\varepsilon_{0}=0.18$, an unstable mode having spiral structures similar to those of $\varepsilon_{0}=0.1$ (figure $23 \mathrm{a}$ ) is found (figure 24). However, the energy ratio is much different; the $m=-1$ and $m=0$ waves have $24.5 \%$ and $51.2 \%$ of the total enery, respectively, while they are $13.1 \%$ and $77.6 \%$ for $\varepsilon_{0}=0.1$ (figure $23 \mathrm{~b}$ ). Since the amplitude of the mode is proportional to the square root of the energy, the amplitude ratio of the $m=-1$ wave to the $m=0$ wave for $\varepsilon_{0}=0.18$ is $\sqrt{0.245 / 0.512}=0.692$ and that for $\varepsilon_{0}=0.1$ is $\sqrt{0.131 / 0.776}=0.411$; thus the amplitude ratio for $\varepsilon_{0}=0.18$ is $0.692 / 0.411=1.68$ times larger than that for $\varepsilon_{0}=0.1$. This ratio is close to that of $\varepsilon_{0}: 1.68 \approx 0.18 / 0.1=1.8$. It suggests that this $m=-1$ wave is not an inertial wave since the amplitude ratio should be independent of $\varepsilon_{0}$ at the leading order for parametric resonance. Instead it can be $O(\varepsilon)$ component created by the $m=0$ wave and the $O(\varepsilon)$ dipole component of the base flow. The growth rate for $\varepsilon_{0}=0.18$ is $\sigma=0.0193$, which is 3.8 times larger than that for $\varepsilon_{0}=0.1$. It suggests that the growth rate of the spiral mode is essentially proportional to $\varepsilon_{0}^{2}$ as the value 3.8 is not far from $(0.18 / 0.1)^{2}=3.24$. It may be explained as the resonance of the $m=0$ wave of $O(1)$ and the $m=-1$ wave of $O(\varepsilon)$ by the $O(\varepsilon)$ dipole field, although further studies are required to understand these spiral modes. 

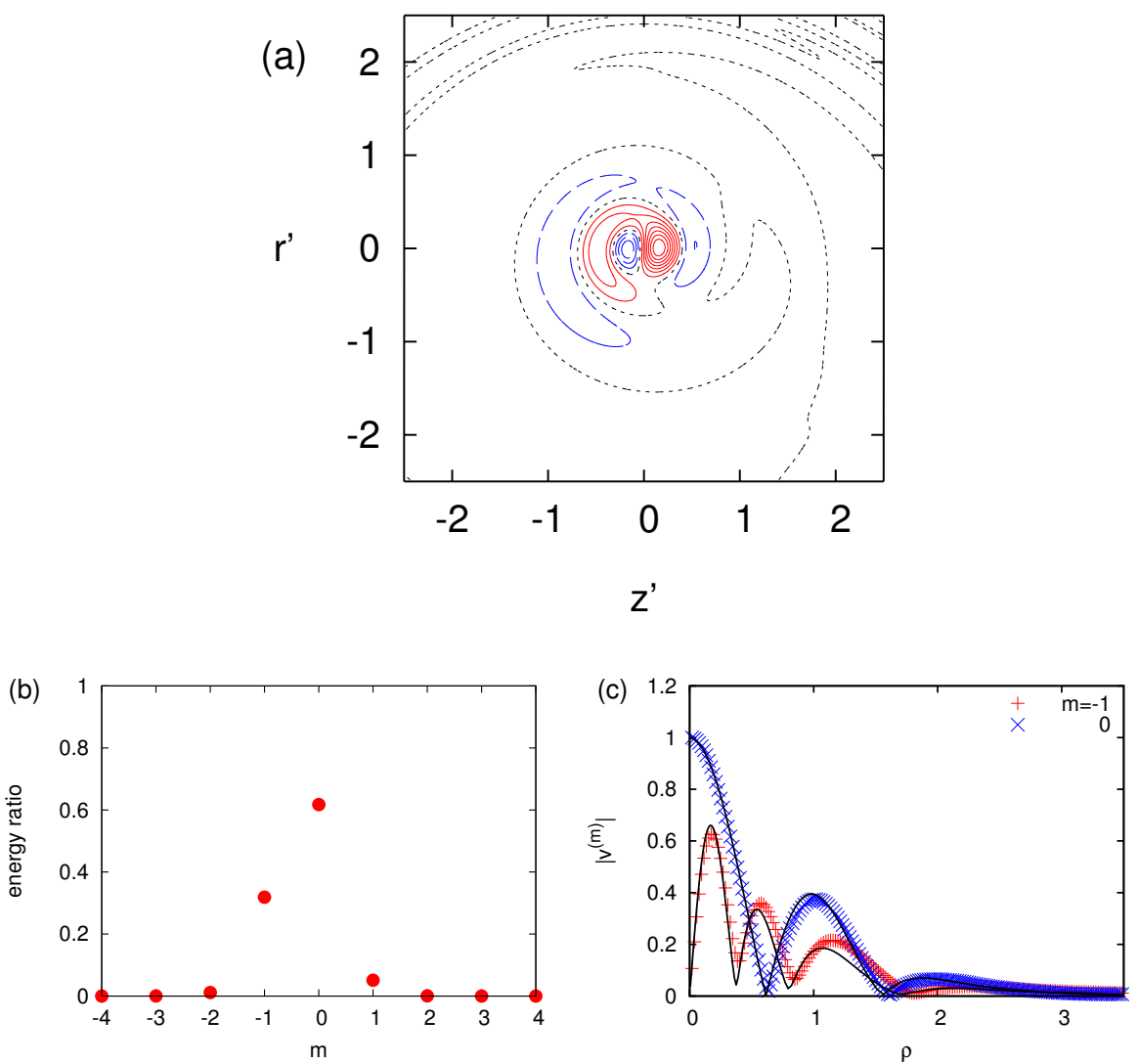

Figure 20. Mode $(-1,0 ;[3,2]), k=1.603, \omega=-0.112$ (curvature instability). $W=0.4, R e=10^{4}, \varepsilon_{0}=0.1$. (a) Contours of azimuthal component of vorticity $\omega_{\theta}: \omega_{\theta}>0$ (solid), $=0$ (dotted) and $<0$ (dashed). (b) Ratio of energy. (c) Radial distribution of $v^{(m)}$; symbols: DNS, lines: theory.

\section{Nonlinear effects}

Although the main focus of the present paper is the linear instability of a vortex ring, it is also of interest and importance to investigate nonlinear time evolution of a disturbed vortex ring. We investigate nonlinear evolution of a vortex ring disturbed by a curvature instability mode focusing on the weakly nonlinear saturation.

The full nonlinear Navier-Stokes equations are solved; the Fourier spectral method is also used in the $\theta$ direction. The curvature instability mode of $(-1,0 ;[2,4])$ with $\varepsilon_{0}=0.18$ for $W=0.2$ is chosen as the initial disturbance. Grid $\mathrm{A}$ in table 2 is used in the $r$ and $z$ directions. We assume $N_{\theta}$-fold symmetry in $\theta$, where $N_{\theta}$ is set to 10 . This implies that subharmonic waves of the initial disturbance are excluded. The number of modes in the $\theta$ direction is 128 . The Reynolds number is set to $R e=5000$ and $10^{4}$, where the former value is used to accommodate the smallest structures that develop at the nonlinear stage. The initial energy of the disturbance is $0.0076 \%$ and $0.0093 \%$ of the base flow energy for $R e=5000$ and $10^{4}$, respectively, while the effects of the initial energy are investigated for the latter case. It is pointed out that as time proceeds the core size of the vortex ring increases and the azimuthal velocity decreases because of viscous diffusion. This implies that the resonance condition of the curvature instability initially satisfied may be broken later. 

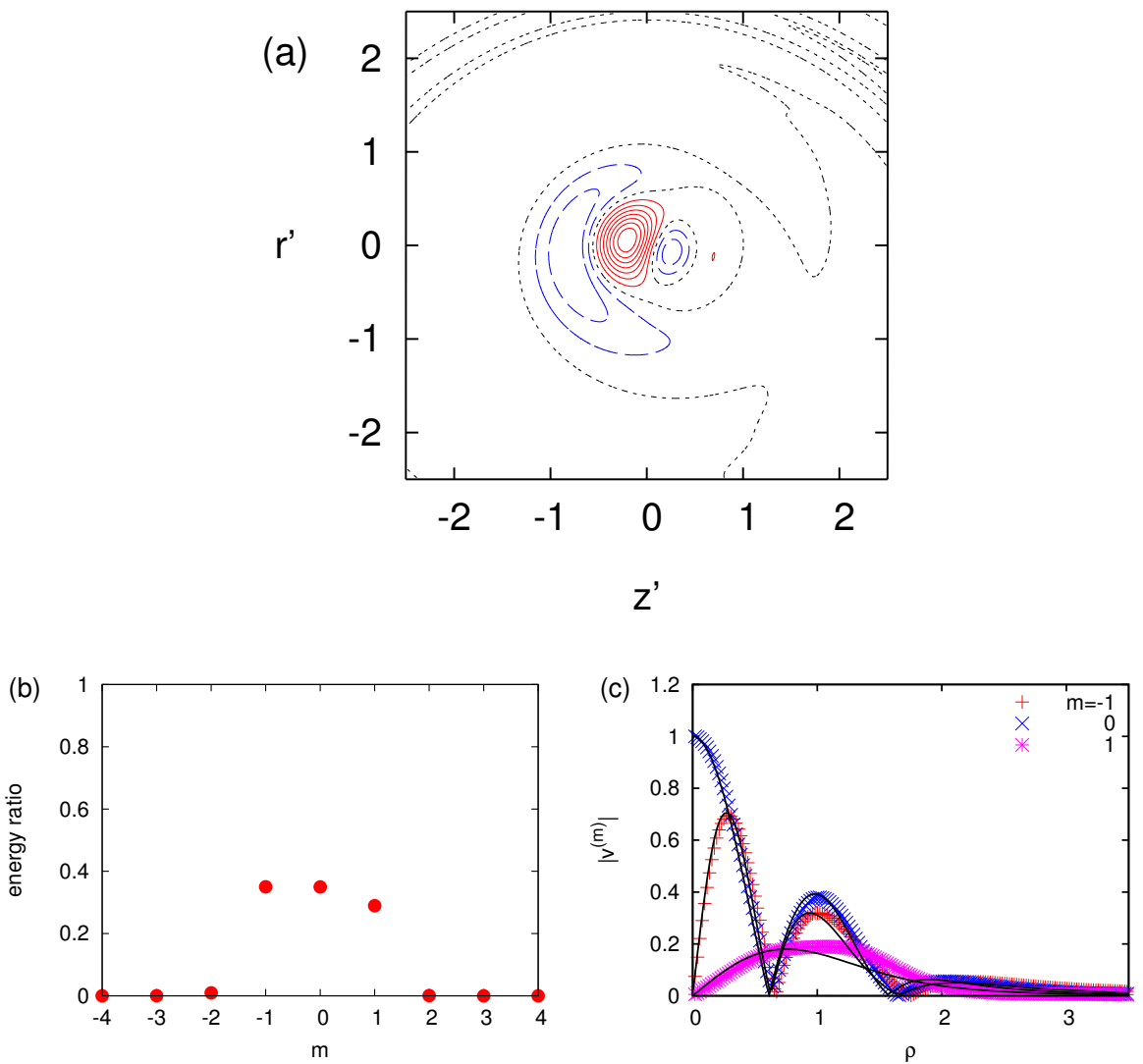

Figure 21. Mode $(-1,0,1 ;[2,2, D]), k=1.389, \omega=-0.106$ (three-wave mode). $W=0.4, R e=10^{4}, \varepsilon_{0}=0.1$. (a) Contours of azimuthal component of vorticity $\omega_{\theta}: \omega_{\theta}>0$ (solid), $=0$ (dotted) and $<0$ (dashed). (b) Ratio of energy. (c) Radial distribution of $v^{(m)}$; symbols: DNS, lines: theory.

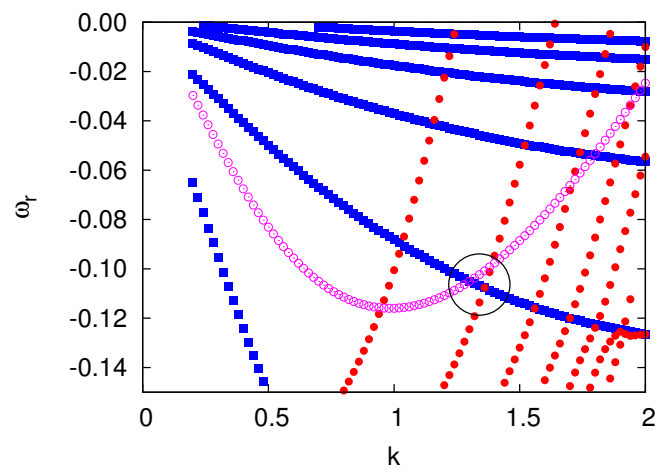

FiguRE 22. Dispersion curves. $R e=10^{4}, W=0.4$. Solid circles: $m=-1$, squares: $m=0$, open circles: $m=1$ (displacement mode). The big circle marks the crossing point of the three-wave mode. 

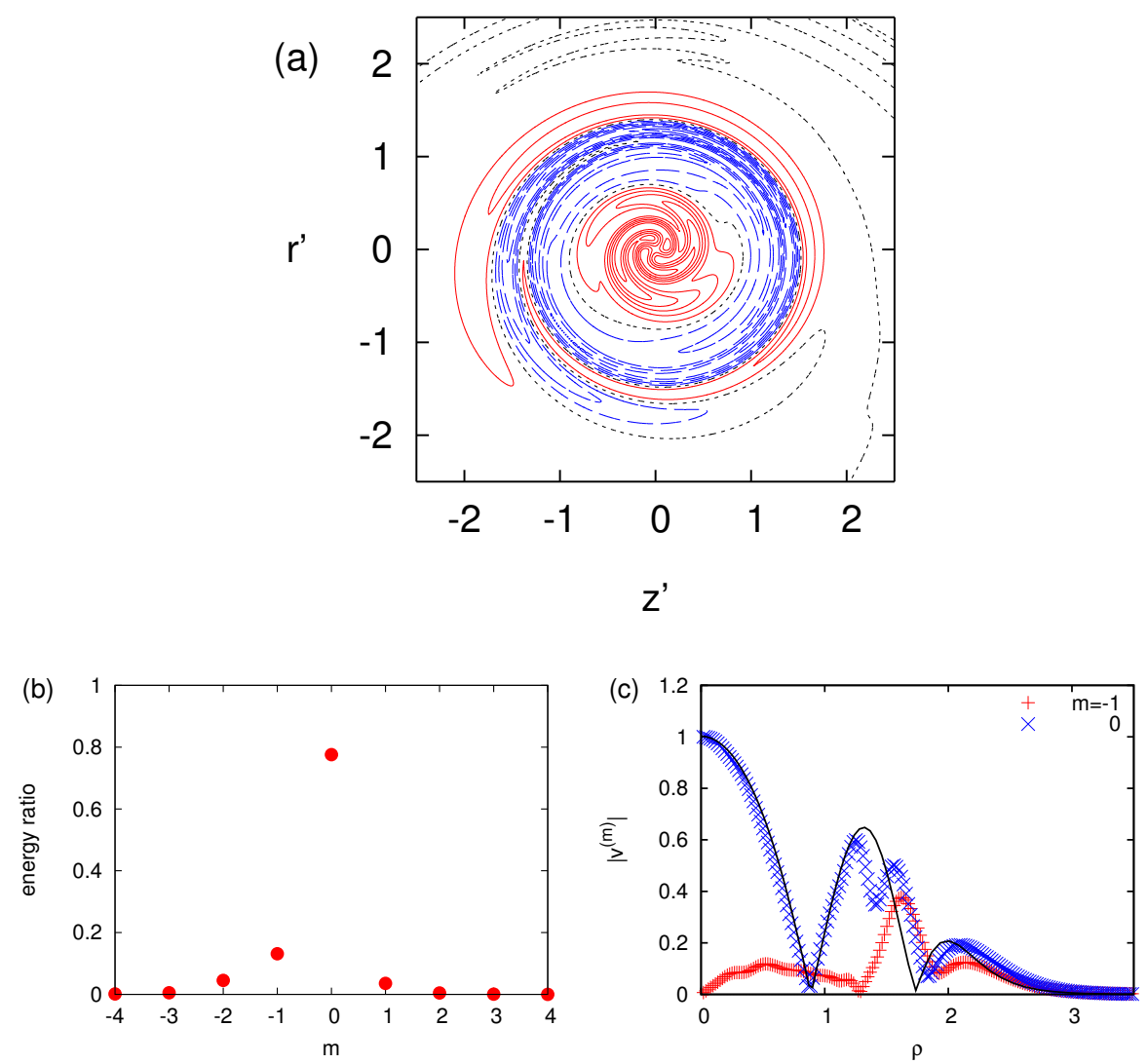

Figure 23. Spiral mode $(-1,0), k=4.231, \omega=-0.078 . W=0.4, R e=10^{4}, \varepsilon_{0}=0.1$. (a) Contours of azimuthal component of vorticity $\omega_{\theta}: \omega_{\theta}>0$ (solid), $=0$ (dotted) and $<0$ (dashed). (b) Ratio of energy. (c) Radial distribution of $v^{(m)}$; symbols: DNS, lines: theory.

Figure 25 shows the time evolution of the disturbance energy decomposed in $\theta: E_{n}=$ $\int\left|\tilde{\boldsymbol{u}}_{n}\right|^{2} r d r d z$, where the velocity field is decomposed as $\boldsymbol{u}=\sum_{n} \tilde{\boldsymbol{u}}_{n}(t, r, z) e^{\mathrm{i} n N_{\theta} \theta}$. At first the curvature instability mode $(n=1)$ grows exponentially. The growth rate is $\sigma=0.00803$ at $t=25$; it is smaller than the observed value of 0.0113 for $R e=10^{4}$ (figure 11) since viscous damping is stronger for $R e=5000$. The higher-order modes $n \geqslant 2$ appear and grow faster than the main disturbance $n=1$. The growth stops at $t \approx 50$. Then the mode energy decreases exponentially, while the $n=1$ mode approaches the initial value at $t \approx 90$. This is a typical behaviour predicted by weakly nonlinear analysis as explained later in some detail. Thus the peak at $t \approx 50$ is due to weakly nonlinear saturation. Figure 26(a) shows the energy ratio of the $n=1$ mode at $t=20$ and 50. It confirms that the instability is due to the curvature instability as the energy ratio is close to that of the $(-1,0 ;[2,4])$ mode shown in figure $12(\mathrm{~b})$. The structures of the whole vortex ring are shown in figure 27. Distortion of the ring is nearly due to the curvature instability mode at $t=25$, while small scale structures are visible at $t=50$.

The mode energy starts growing again at $t \approx 95$. The exponential growth rate is larger than that of the initial growth. It is due to the elliptic instability as shown below. Figure 26 (b) shows the energy ratio of the $n=1$ mode at $t=120$. It consists of $m= \pm 1$ waves. This elliptic instability mode corresponds to $(-1,1 ;[1,1])$, which is shown in figure 8 for $W=0$. Although the core size and the swirl parameter are set to $\varepsilon=0.18$ and $W=0.2$, 

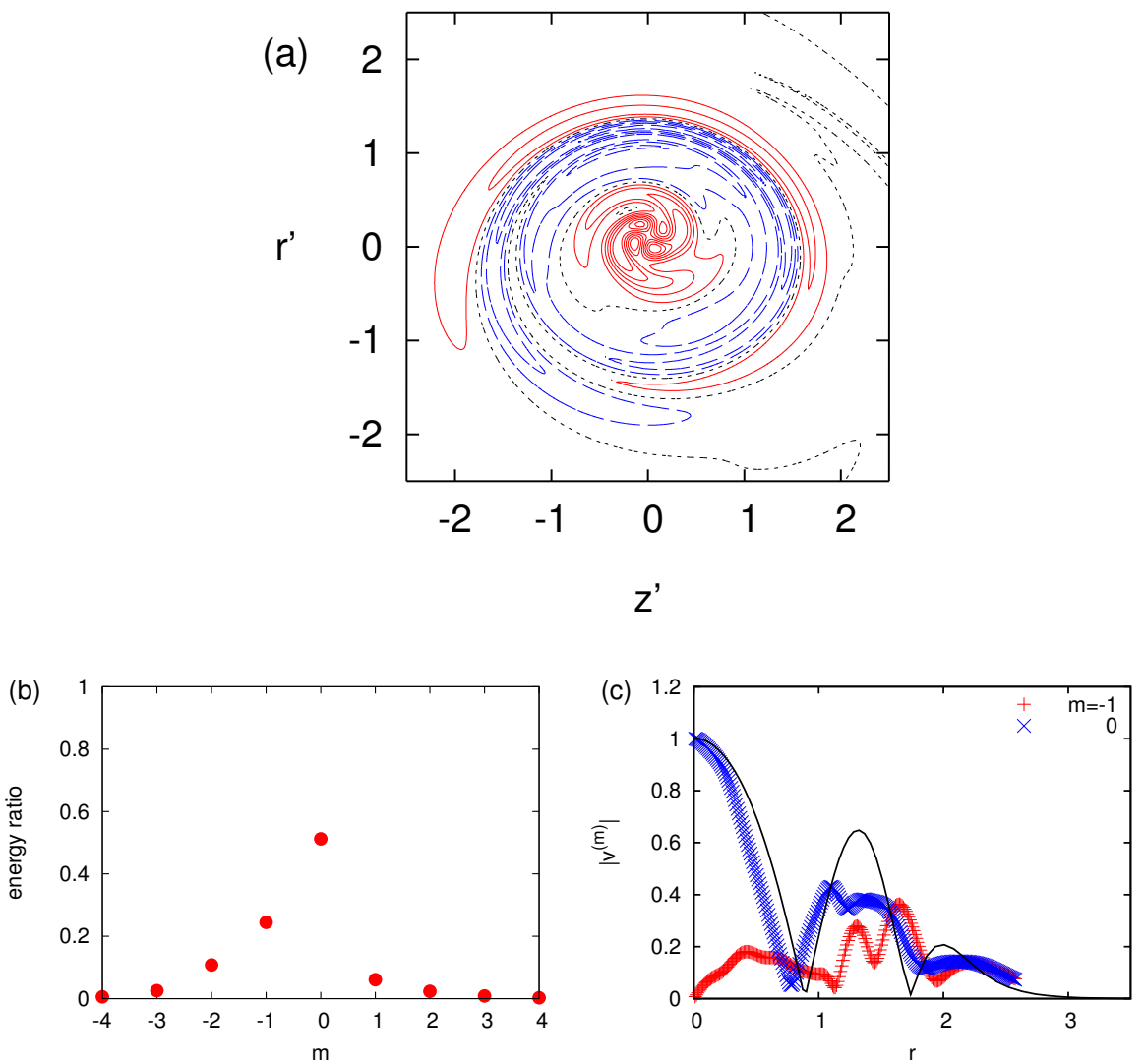

Figure 24. Spiral mode $(-1,0), k=4.231, \omega=-0.078 . W=0.4, R e=10^{4}, \varepsilon_{0}=0.18$. (a) Contours of azimuthal component of vorticity $\omega_{\theta}: \omega_{\theta}>0$ (solid), $=0$ (dotted) and $<0$ (dashed). (b) Ratio of energy. (c) Radial distribution of $v^{(m)}$; symbols: DNS, lines: theory.

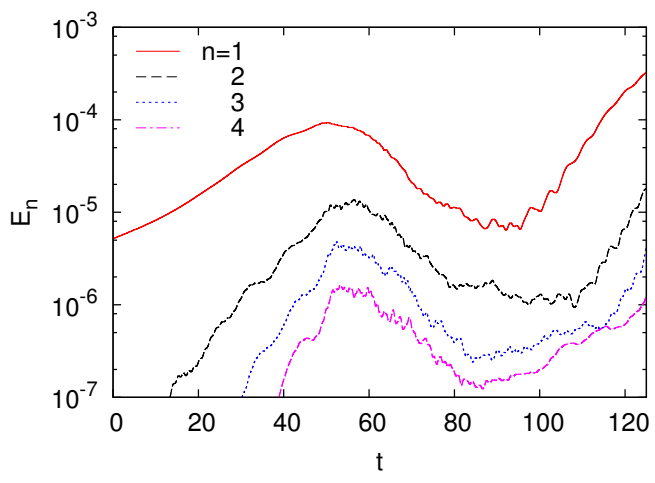

FIGURE 25. Time evolution of the disturbance energy obtained by nonlinear simulation. The disturbance energy is decomposed in $\theta: E_{n}=\int\left|\tilde{\boldsymbol{u}}_{n}\right|^{2} r d r d z$, where the velocity field is decomposed as $\boldsymbol{u}=\sum_{n} \tilde{\boldsymbol{u}}_{n}(t, r, z) e^{\mathrm{i} n N_{\theta} \theta}$. 

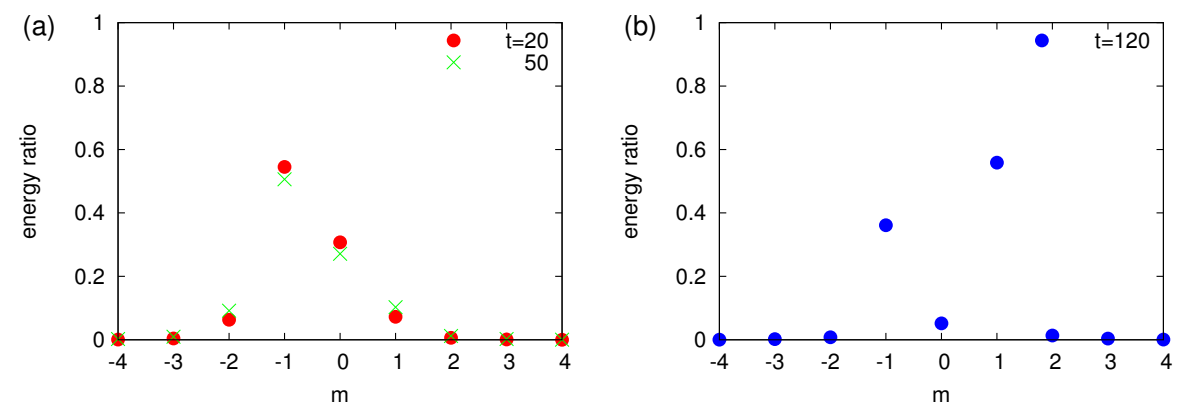

FiguRE 26. Ratio of energy of the linear mode $\tilde{\boldsymbol{u}}_{1}(t, r, z)$ obtained by nonlinear simulation. The linear mode is decomposed in the local polar coordinate system as in (4.1). (a) $t=20,50$, (b) $t=120$.
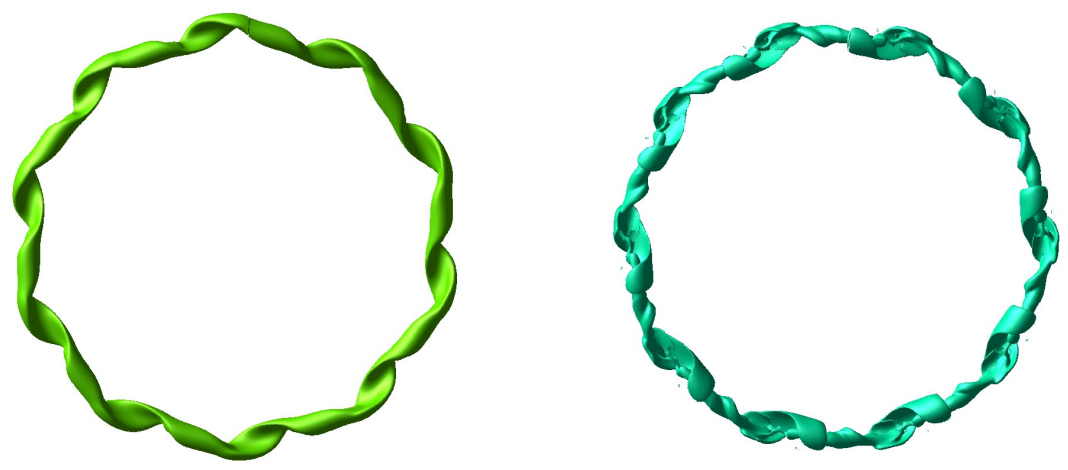

FIGURE 27. Structure of disturbed vortex ring obtained by nonlinear simulation. The isosurface of the magnitude of vorticity is shown. (Left) $t=25$, (right) $t=50$.

respectively, at $t=0$, they become $\varepsilon \approx 0.3$ and $W \approx 0.12$, respectively, at $t=120$ (figure 31 ); as a result the resonance condition for parametric instability moves from the curvature instability mode $(-1,0 ;[2,4])$ to the elliptic instability mode $(-1,1 ;[1,1])$.

Effects of the initial amplitude of the disturbance are investigated for $R e=10^{4}$ to clarify how nonlinearity leads to saturation of the curvature instability. Figure 28 compares time evolution of the energy of the linear mode $E_{1}$ for different values of the initial amplitude $A / A_{0}=1,10^{-1}, \cdots, 10^{-4}$. Figure $28(\mathrm{a})$ shows that $E_{1}$ saturates at $t=40.9,121.2$ and 189.1 for $A / A_{0}=1,10^{-1}$ and $10^{-2}$, respectively. The fact that $E_{1} \approx 10^{-4}$ does not depend on the initial amplitude very much supports that the saturation is due to weakly nonlinear effects. On the other hand, the elliptic instability takes over the curvature instability at $t \approx 200$ for $A / A_{0}=10^{-3}$ and $10^{-4}$ before saturation. In figure $28(\mathrm{~b})$ the time evolutions of $E_{1}$ are divided by $\left(A / A_{0}\right)^{2}$ so that they collapse at $t \approx 0$. The time evolution starts to deviate from those for lower initial amplitudes when nonlinear effects become important. This shows that nonlinearity starts to affect the dynamics at $t \approx 20,90$ and 170 for $A / A_{0}=1,10^{-1}$ and $10^{-2}$, which are $20 \sim 30$ before the saturation.

Figure 29 shows time evolution of the vortex ring by distribution of the $\theta$ component of vorticity on a cross section $(\theta=0)$. The total vorticity $\omega_{\theta}$ (top row), the mean 

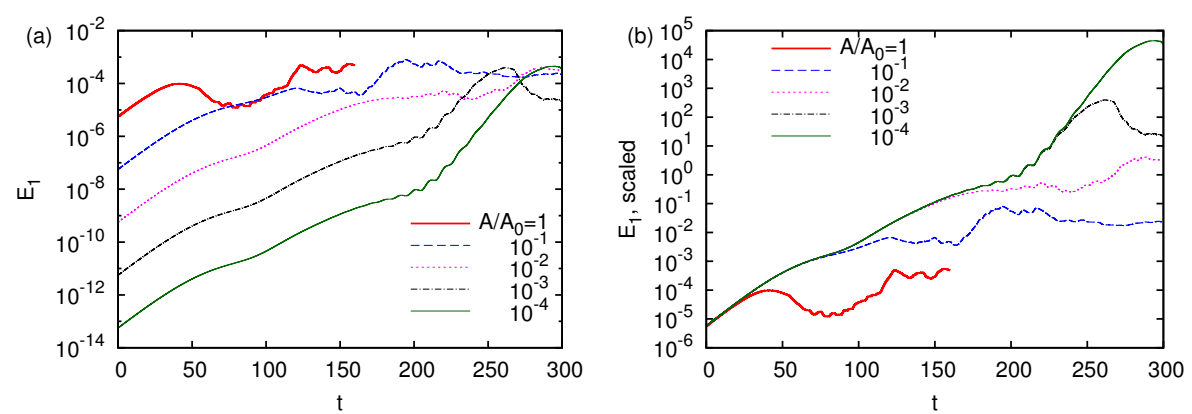

Figure 28. Time evolution of the disturbance energy obtained by nonlinear simulation. $R e=10^{4}$. (a) The disturbance energy of the linear mode $E_{1}$ is compared for different values of the initial disturbance energy. (b) Same as (a) but the values are scaled to collapse at $t=0$.

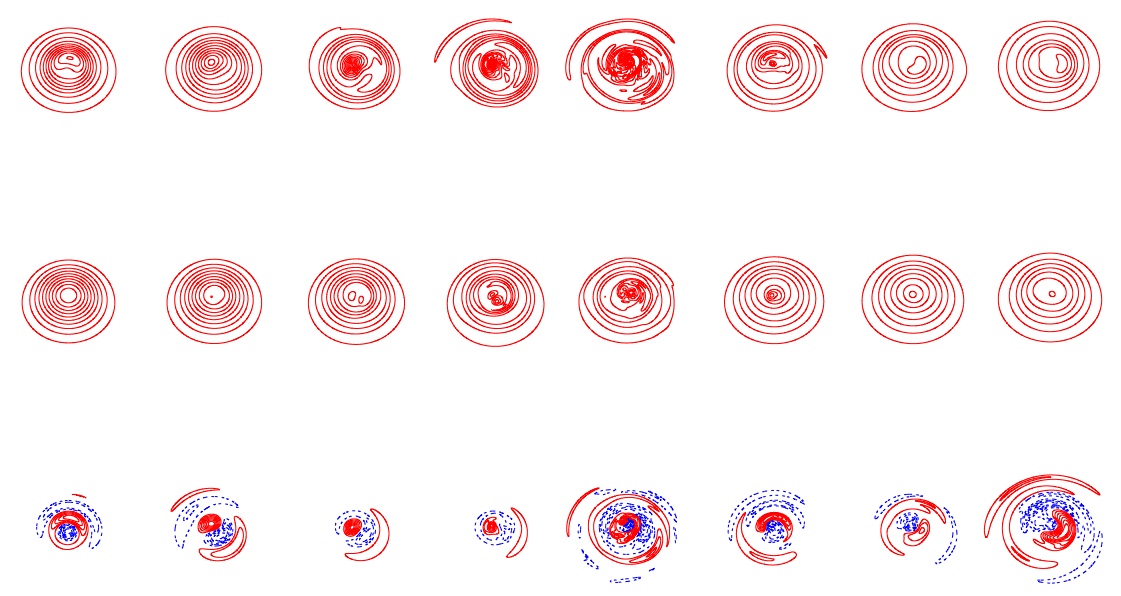

Figure 29. Time evolution of disturbed vortex ring. Contours of $\theta$ component of vorticity on $\theta=0$ are shown. $R e=5000$. (Top row) total vorticity $\omega_{\theta}$, (middle row) mean vorticity $\tilde{\omega}_{\theta, 0}$, (bottom row) disturbance vorticity $\omega_{\theta}-\tilde{\omega}_{\theta, 0}$. From left to right: $t=0,16,32,48,64,80,96,112$. Contour levels are $n \Delta \omega(n= \pm 1, \pm 2, \cdots)$ while $\Delta \omega=1$ for the total and mean vorticity and $\Delta \omega=\left|\omega_{\theta}-\tilde{\omega}_{\theta, 0}\right|_{\max } / 8$ for the disturbance vorticity. Positive and negative values are shown by red and blue lines, respectively.

vorticity $\tilde{\omega}_{\theta, 0}$ (middle row) and the disturbance vorticity $\omega_{\theta}-\tilde{\omega}_{\theta, 0}$ (bottom row) are shown for $t=0,16,32,48,64,80,96$ and 112. It is pointed out that the contour levels are fixed for the total and mean vorticity, while they depend on the maximum value for the disturbance vorticity since the magnitude varies significantly with time. The total vorticity is significantly disturbed at $t=32,48$ and 64 when the disturbance energy is large, while it goes back to smooth state at $t=96$ and 112 . The same trend is also observed for the mean vorticity with lower level of disturbance. The distribution of the disturbance vorticity does not differ much from the curvature instability mode of $(-1,0 ;[2,4])$ up to $t=48$. However, the distribution changes after $t=48$; at $t=96$ and 112 it shows structures of bending wave due to the elliptic instability.

The details of weakly nonlinear saturation can be shown by the phases of the $m=$ -1 and $m=0$ waves which compose the curvature instability mode. Figure 30 shows 

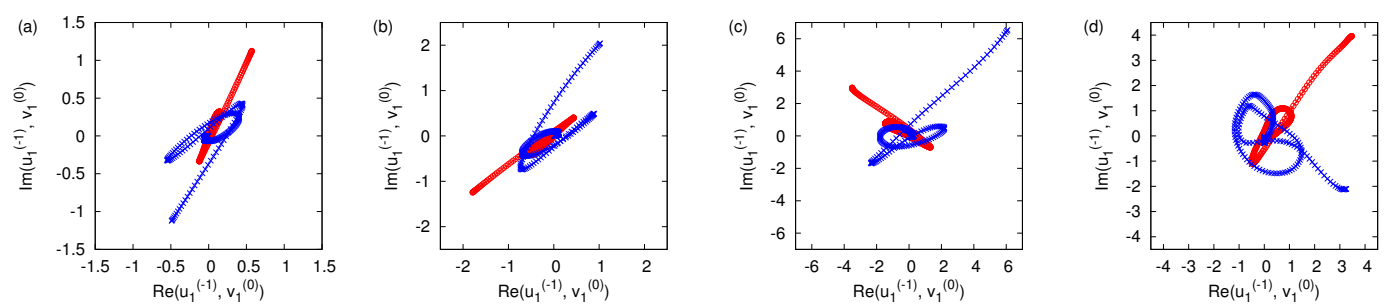

FigURE 30. Linear modes on the complex plane. The linear modes are decomposed in the local coordinate system $(\rho, \phi)$ and $\tilde{u}_{1}^{(-1)}$ (shown by red circles) and $\tilde{v}_{1}^{(0)}$ (shown by blue crosses) are plotted on the complex plane. $R e=5000$. (a) $t=0$, (b) $t=20$, (c) $t=48$, (d) $t=60$.

those waves on the complex plane for $t=0,20,48$ and 60 . The linear mode $\tilde{\boldsymbol{u}}_{1}(t, r, z)$ is decomposed in the local coordinate system (figure 1) and $\tilde{u}_{1}^{(-1)}$ and $\tilde{v}_{1}^{(0)}$ are shown by red circles and blue crosses, respectively. The curves rotate with time since the two waves are oscillatory. The curves at $t=0$ and $t=20$ are similar in shape after rotation, while they deform gradually as time proceeds $(t=48$ and 60$)$. The phase difference between $\tilde{u}_{1}^{(-1)}$ and $\tilde{v}_{1}^{(0)}$ is important here. We take the phase at maximum amplitude for each mode. Then the phase difference is $-177^{\circ},-151^{\circ},-93^{\circ}$ and $-82^{\circ}$ at $t=0,20,48$ and 60 , respectively. The value of phase difference depends on the section or $\theta$. An important point is that the phase difference increases with $84^{\circ} \approx \pi / 2$ from the initial state $t=0$ to $t=48$ at which the curvature instability saturates. This change in the phase difference is explained in terms of the amplitude equation derived by the weakly nonlinear analysis. In the simplest form, the amplitude equation is

$$
\begin{aligned}
\frac{\mathrm{d} A_{-1}}{\mathrm{~d} t} & =c_{1} A_{0}+\mathrm{i}\left(s_{1}\left|A_{-1}\right|^{2}+s_{2}\left|A_{0}\right|^{2}\right) A_{-1}, \\
\frac{\mathrm{d} A_{0}}{\mathrm{~d} t} & =c_{2} A_{-1}+\mathrm{i}\left(s_{3}\left|A_{-1}\right|^{2}+s_{4}\left|A_{0}\right|^{2}\right) A_{0},
\end{aligned}
$$

where $A_{-1}$ and $A_{0}$ are the complex amplitudes of the $m=-1$ and $m=0$ waves and $c_{i}$ and $s_{i}$ are real constants satisfying $c_{1} c_{2}>0$ (Knobloch, Mahalov \& Marsden 1994). At the linear stage $\left|A_{-1}\right|$ and $\left|A_{0}\right|$ are sufficiently small so that the nonlinear terms are negligible; then the phase difference between $A_{-1}$ and $A_{0}$ is 0 for the exponentially growing solution to equations (5.1) and (5.2). On the other hand, when the growth saturates which implies $d A_{-1} / d t=d A_{0} / d t=0$, the phase difference between $A_{-1}$ and $A_{0}$ is $\pi / 2$ according to equations (5.1) and (5.2); in other words, the detuning effects of nonlinear terms stop the curvature instability. Thus the phase difference increases with $\pi / 2$ when the growth saturates. Therefore, the change in the phase difference observed in figure 30 is a result of weakly nonlinear saturation described by the amplitude equation (Sipp 2000; Eloy, Le Gal \& Le Dizès 2000, 2003).

Figure 31 shows time evolution of characteristic values of the vortex ring: the core radius, the swirl parameter $W$, the maximum vorticity, the circulation and the energy. Here the values are normalized by the initial values except for the core radius and the energy is plotted with its square root to be proportional to the velocity for comparison. The figures show that the swirl parameter increases gradually before nonlinear saturation; then it drops at $48 \lesssim t \lesssim 64$. Correspondingly, the maximum vorticity decreases before nonlinear saturation; then it increases at $48 \lesssim t \lesssim 70$. Thus there are changes in the mean flow after the nonlinear saturation. The viscous spreading of the core is slightly accelerated after the saturation (figure 31a), although the circulation is nearly constant up to $t \approx 80$ and the decay of the energy is unaffected up to $t \approx 120$ (figure $31 \mathrm{c}$ ). 

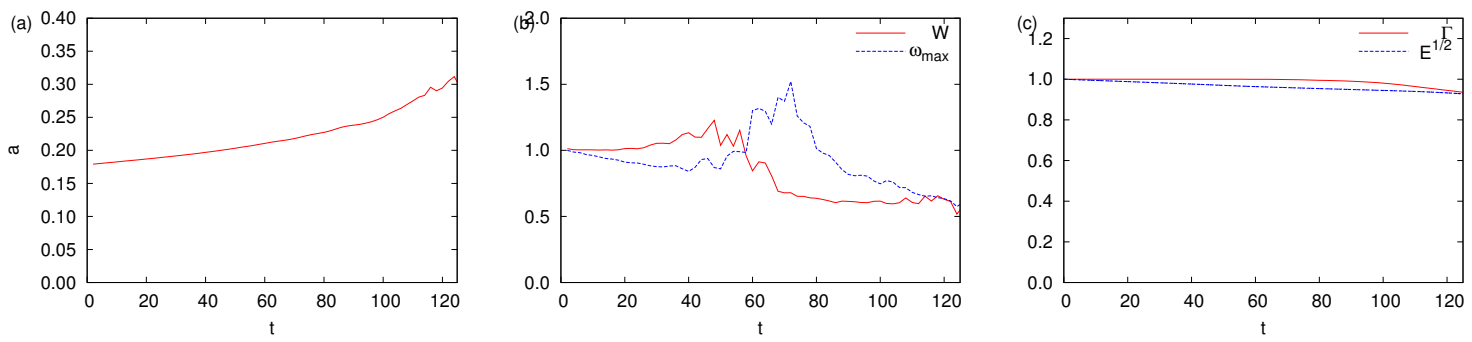

Figure 31. Time evolution of characteristic values of the vortex ring. $R e=5000$. (a) Core radius, (b) swirl parameter and maximum vorticity, (c) circulation and square root of energy. Note that the values are normalized by the initial values for comparison.

To summarize, the curvature instability grows until weakly nonlinear saturation; after the saturation nonlinear effects modify the mean flow decreasing the swirl parameter and the disturbance energy decreases. It is pointed out that the secondary instability following the curvature instability is not observed.

\section{Concluding remarks}

We have studied the linear instability of a vortex ring with swirl with Gaussian distributions of azimuthal vorticity and velocity in its core at the leading order of the thickness by direct numerical simulation: the quasi-steady base flow was obtained by solving the axisymmetric incompressible Navier-Stokes equations; then the linearized Navier-Stokes equations were solved by DNS. It was shown that the vortex rings are subjected to the curvature instability as predicted analytically by Blanco-Rodríguez \& Le Dizès (2017). The structures of the unstable modes obtained numerically are in good agreement with the analytical results. For the growth rates, on the other hand, some differences between numerical and analytical results were found depending on the unstable mode. The differences are most likely due to the critical layer damping which is evaluated for the waves on axisymmetric line vortices in the analysis; the actual growth rate is affected by the deformation of the core since the damping is sensitive to the position of the critical layer. Competition between the curvature and elliptic instabilities was also investigated. When the swirl is absent, only the elliptic instability is observed since the growth rate of the curvature instability is small. When the swirl is added, sharp bands of the two instabilities are observed for small thickness $\varepsilon \approx 0.1$, while the elliptic instability dominates except for a small interval of the wavenumber for $\varepsilon \approx 0.18$. A special mode which consists of three waves was also found; although it is essentially due to the curvature instability mode of $(-1,0 ;[2,2])$, a displacement wave of $m=1$ happens to be close to the corresponding crossing point of the dispersion curves and joins the unstable mode. Spiral modes which may be due to $O\left(\varepsilon^{2}\right)$ effects were also found. Weakly nonlinear saturation has been shown to occur by nonlinear simulation.

It is emphasized that the curvature instability has been shown by direct numerical simulation for the first time; although by theoretical analysis it was first shown for Kelvin's vortex ring (Fukumoto \& Hattori 2005; Hattori \& Fukumoto 2003) and then for a Gaussian vortex ring (Blanco-Rodríguez \& Le Dizès 2017), no results by numerical simulation or experiments have been available before the present work. The present results should be taken into account carefully in interpreting the experimental results since the curvature instability can be the dominant instability for large Reynolds numbers and small thickness. In the numerical work by Gargan-Shingles, Rudman \& Ryan (2016) 
the Reynolds number is smaller than the the present work: $R e=10^{4} /(2 \pi)=1592$. In addition, the thickness is larger: $a / R=0.234 \sim 0.344$. These are the reasons why they did not observe the curvature instability, although the mode shown in Fig. 9A of GarganShingles, Rudman \& Ryan (2016) may be due to curvature instability. Another result worth emphasizing is that the critical layer damping is sensitive to the deformation of the vortex core; the growth rate of the curvature instability mode $(-1,0 ;[1,3])$ at $\varepsilon_{0}=0.1$ was found to be $22 \%$ smaller than the theoretical value, while it is in good agreement for the mode $(-1,0 ;[2,4])$. This difference is most likely due to the critical layer damping which is strong for the former but weak for the latter. This result is important not only for the curvature instability but also for any short-wave instability due to parametric resonance like elliptic instability and precession instability which occurs in a precessing cylinder (Kerswell 1993; Mahalov 1993; Lagrange, Eloy, Nadal \& Meunier 2008).

Finally, two important future works are mentioned briefly below. First, nonlinear evolution of curvature instability modes should be investigated further to clarify their role in the dynamics of a vortex ring; it can lead to the breakdown of a vortex ring. Next, the curvature instability of a helical vortex should be explored; as shown theoretically by Hattori \& Fukumoto (2009, 2010, 2012, 2014) and Blanco-Rodríguez \& Le Dizès (2017) helical vortices are subjected to the curvature instability in general. It is of great importance since the helical vortices are a model of tip vortices generated by rotating wings like helicopter rotors, wind rotors and ship propellers. The curvature instability is expected to be important as the conditions for the curvature instability to be dominant are often satisfied for these rotating wings: the Reynolds number is large, the thickness is small and the base flow has a non-vanishing swirl. The numerical methods developed in Delbende, Rossi, \& Daube (2012) and Selçuk, Delbende \& Rossi (2018) would be useful for this problem.

This work was supported by Collaborative Research Project 2016-2018, Institute of Fluid Science, Tohoku University. This work also received support from the French Agence Nationale de la Recherche under the A*MIDEX grant ANR-11-IDEX-0001-02, the LABEX MEC project ANR-11-LABX-0092 and the ANR HELIX project ANR12-BS09-0023-01. Y.H. is grateful to IRPHE for the kind hospitality during his visit. Numerical calculations were performed on the UV2000 at the Institute of Fluid Science, Tohoku University.

\section{Appendix A. Details of numerical methods}

In this appendix we describe the details of our numerical method.

The incompressible Navier-Stokes equations are written as

$$
\begin{aligned}
\frac{\partial u}{\partial t}= & -u \frac{\partial u}{\partial r}-\frac{v}{r} \frac{\partial u}{\partial \theta}-w \frac{\partial u}{\partial z}+\frac{v^{2}}{r}-\frac{\partial p}{\partial r}+\nu\left[\left(\nabla^{2}-\frac{1}{r^{2}}\right) u-\frac{2}{r^{2}} \frac{\partial v}{\partial \theta}\right], \\
\frac{\partial v}{\partial t}= & -u \frac{\partial v}{\partial r}-\frac{v}{r} \frac{\partial v}{\partial \theta}-w \frac{\partial v}{\partial z}-\frac{u v}{r}-\frac{1}{r} \frac{\partial p}{\partial \theta}+\nu\left[\left(\nabla^{2}-\frac{1}{r^{2}}\right) v+\frac{2}{r^{2}} \frac{\partial u}{\partial \theta}\right], \\
\frac{\partial w}{\partial t}= & -u \frac{\partial w}{\partial r}-\frac{v}{r} \frac{\partial w}{\partial \theta}-w \frac{\partial w}{\partial z}-\frac{\partial p}{\partial z}+\nu \nabla^{2} w \\
& \frac{\partial u}{\partial r}+\frac{u}{r}+\frac{1}{r} \frac{\partial v}{\partial \theta}+\frac{\partial w}{\partial z}=0
\end{aligned}
$$

where $u, v$ and $w$ are the $r, \theta$ and $z$ components of the velocity and $\nabla^{2}=\frac{\partial^{2}}{\partial r^{2}}+\frac{1}{r} \frac{\partial}{\partial r}+$ $\frac{1}{r^{2}} \frac{\partial^{2}}{\partial \theta^{2}}+\frac{\partial^{2}}{\partial z^{2}}$. The above equations are discretized in time with the second-order implicit 
method for the viscous terms and the Adams-Bashforth method for the other terms

$$
\begin{aligned}
& {\left[\frac{3}{2}-\nu \Delta t \nabla^{2}\right] u^{(n+1)}=2 u^{(n)}-\frac{1}{2} u^{(n-1)}+\frac{\Delta t}{2}\left(3 H_{u}^{(n)}-H_{u}^{(n-1)}\right),} \\
& {\left[\frac{3}{2}-\nu \Delta t \nabla^{2}\right] v^{(n+1)}=2 v^{(n)}-\frac{1}{2} v^{(n-1)}+\frac{\Delta t}{2}\left(3 H_{v}^{(n)}-H_{v}^{(n-1)}\right),} \\
& {\left[\frac{3}{2}-\nu \Delta t \nabla^{2}\right] w^{(n+1)}=2 w^{(n)}-\frac{1}{2} w^{(n-1)}+\frac{\Delta t}{2}\left(3 H_{w}^{(n)}-H_{w}^{(n-1)}\right),}
\end{aligned}
$$

where $f^{(n)}$ denotes $f$ at the $n$-th time step,

$$
\begin{aligned}
& H_{u}=h_{u}-\frac{\partial \Phi}{\partial r} \\
& H_{v}=h_{v}-\frac{1}{r} \frac{\partial \Phi}{\partial \theta}, \\
& H_{w}=h_{w}-\frac{\partial \Phi}{\partial z},
\end{aligned}
$$

and

$$
\begin{aligned}
& h_{u}=-u \frac{\partial u}{\partial r}-\frac{v}{r} \frac{\partial u}{\partial \theta}-w \frac{\partial u}{\partial z}+\frac{v^{2}}{r}+\nu\left(-\frac{u}{r^{2}}-\frac{2}{r^{2}} \frac{\partial v}{\partial \theta}\right), \\
& h_{v}=-u \frac{\partial v}{\partial r}-\frac{v}{r} \frac{\partial v}{\partial \theta}-w \frac{\partial v}{\partial z}-\frac{u v}{r}+\nu\left(-\frac{v}{r^{2}}+\frac{2}{r^{2}} \frac{\partial u}{\partial \theta}\right), \\
& h_{w}=-u \frac{\partial w}{\partial r}-\frac{v}{r} \frac{\partial w}{\partial \theta}-w \frac{\partial w}{\partial z} .
\end{aligned}
$$

It should be pointed out that the cross terms in the viscous terms (e.g. $\nu \frac{2}{r^{2}} \frac{\partial v}{\partial \theta}$ in (A 11)) are moved to the righthand side together with the corresponding terms which cancel their divergent behaviour near $r=0$ (e.g. $\nu \frac{u}{r^{2}}$ in (A 11)) as only the Laplacian terms are dealt with implicitly; this simplifies the numerical procedure. The function $\Phi$ is obtained by solving the Poisson equation

$$
\nabla^{2} \Phi=\frac{\partial h_{u}}{\partial r}+\frac{h_{u}}{r}+\frac{1}{r} \frac{\partial h_{v}}{\partial \theta}+\frac{\partial h_{w}}{\partial z}
$$

The boundary conditions at $r= \pm L_{r}$ are

$$
u=0, \quad \frac{\partial}{\partial r}(r v)=\frac{\partial}{\partial r} w=0 .
$$

The velocity fields expanded in Fourier series as $f=\sum_{n} \hat{f}_{m, n}(r, t) e^{\mathrm{i}(m \theta+n \alpha z)}$, where $m=0$ for the base flow and $m=N_{\theta}$ for the disturbance and $\alpha=2 \pi / L_{z}$. Then the equations in the Fourier space are

$$
\begin{aligned}
& {\left[\frac{3}{2}-\nu \Delta t\left\{\frac{\mathrm{d}^{2}}{\mathrm{~d} r^{2}}+\frac{1}{r} \frac{\mathrm{d}}{\mathrm{d} r}-\left(\frac{(|m|-1)^{2}}{r^{2}}+\alpha^{2} n^{2}\right)\right\}\right] \hat{u}_{m, n}^{(n+1)}} \\
& =2 \hat{u}_{m, n}^{(n)}-\frac{1}{2} \hat{u}_{m, n}^{(n-1)}+\frac{\Delta t}{2}\left(3 \hat{H}_{u ; m, n}^{(n)}-\hat{H}_{u ; m, n}^{(n-1)}\right), \\
& {\left[\frac{3}{2}-\nu \Delta t\left\{\frac{\mathrm{d}^{2}}{\mathrm{~d} r^{2}}+\frac{1}{r} \frac{\mathrm{d}}{\mathrm{d} r}-\left(\frac{(|m|-1)^{2}}{r^{2}}+\alpha^{2} n^{2}\right)\right\}\right] \hat{v}_{m, n}^{(n+1)}} \\
& =2 \hat{v}_{m, n}^{(n)}-\frac{1}{2} \hat{v}_{m, n}^{(n-1)}+\frac{\Delta t}{2}\left(3 \hat{H}_{v ; m, n}^{(n)}-\hat{H}_{v ; m, n}^{(n-1)}\right), \\
& {\left[\frac{3}{2}-\nu \Delta t\left\{\frac{\mathrm{d}^{2}}{\mathrm{~d} r^{2}}+\frac{1}{r} \frac{\mathrm{d}}{\mathrm{d} r}-\left(\frac{m^{2}}{r^{2}}+\alpha^{2} n^{2}\right)\right\}\right] \hat{w}_{m, n}^{(n+1)}}
\end{aligned}
$$




\begin{tabular}{ccccccc}
\hline case & $\Delta r_{\min } / R_{0}$ & $\Delta z / R_{0}$ & $\Omega_{\max }^{(0)} \Delta t$ & $T_{0}$ & $L_{r} / R_{0}$ & $\sigma$ \\
\hline reference & $2.5 \times 10^{-3}$ & $2.05 \times 10^{-3}$ & $3.51 \times 10^{-3}$ & 12 & 100 & $4.2443 \times 10^{-3}$ \\
case G & $5 \times 10^{-3}$ & $4.09 \times 10^{-3}$ & $7.03 \times 10^{-3}$ & 12 & 100 & $4.2443 \times 10^{-3}$ \\
case L1 & $5 \times 10^{-3}$ & $4.09 \times 10^{-3}$ & $7.03 \times 10^{-3}$ & 12 & 50 & $4.2443 \times 10^{-3}$ \\
case L2 & $5 \times 10^{-3}$ & $4.09 \times 10^{-3}$ & $7.03 \times 10^{-3}$ & 12 & 25 & $4.2443 \times 10^{-3}$ \\
case L3 & $5 \times 10^{-3}$ & $4.09 \times 10^{-3}$ & $7.03 \times 10^{-3}$ & 12 & 10 & $4.2443 \times 10^{-3}$ \\
case DT & $5 \times 10^{-3}$ & $4.09 \times 10^{-3}$ & $3.51 \times 10^{-3}$ & 12 & 100 & $4.2437 \times 10^{-3}$ \\
case TL & $2.5 \times 10^{-3}$ & $2.05 \times 10^{-3}$ & $3.51 \times 10^{-3}$ & 24 & 100 & $4.2125 \times 10^{-3}$ \\
\hline
\end{tabular}

TABLE 4 . Growth rate obtained by different numerical settings. The curvature instability mode of $(-1,0 ;[1,3])$ with $\varepsilon_{0}=0.1$ and $k=1.144$.

$$
=2 \hat{w}_{m, n}^{(n)}-\frac{1}{2} \hat{w}_{m, n}^{(n-1)}+\frac{\Delta t}{2}\left(3 \hat{H}_{w ; m, n}^{(n)}-\hat{H}_{w ; m, n}^{(n-1)}\right) .
$$

The boundary conditions (A 15) become

$$
\hat{u}_{m, n}=0, \quad \frac{\partial}{\partial r}\left(r \hat{v}_{m, n}\right)=\frac{\partial \hat{w}_{m, n}}{\partial r}=0 .
$$

The Poisson equation for $\Phi$ is also expressed in the Fourier space as

$$
\begin{aligned}
& \left\{\frac{\mathrm{d}^{2}}{\mathrm{~d} r^{2}}+\frac{1}{r} \frac{\mathrm{d}}{\mathrm{d} r}-\left(\frac{m^{2}}{r^{2}}+\alpha^{2} n^{2}\right)\right\} \hat{\Phi}_{m, n} \\
& =\frac{\mathrm{d} \hat{h}_{u ; m, n}}{\mathrm{~d} r}+\frac{\hat{h}_{u ; m, n}}{r}+\frac{\mathrm{i} m}{r} \hat{h}_{v ; m, n}+\mathrm{i} \alpha n \hat{h}_{w ; m, n}
\end{aligned}
$$

with the boundary condition

$$
\frac{\partial \hat{\Phi}_{m, n}}{\partial r}=0 .
$$

The equations (A 16)-(A 18) and (A 19) for each Fourier mode are second-order ordinary differential equations; they are solved by sixth-order accurate compact scheme.

The numerical method for the linear stability analysis is same except that the nonlinear terms in $h_{u}, h_{v}$ and $h_{w}$ are replaced by the corresponding linearized terms. For example, $h_{u}$ is replaced by

$$
\begin{aligned}
h_{u}^{\prime}= & -u_{b} \frac{\partial u^{\prime}}{\partial r}-\frac{v_{b}}{r} \frac{\partial u^{\prime}}{\partial \theta}-w_{b} \frac{\partial u^{\prime}}{\partial z}-u^{\prime} \frac{\partial u_{b}}{\partial r}-w^{\prime} \frac{\partial u_{b}}{\partial z}+\frac{2 v_{b} v^{\prime}}{r} \\
& +\nu\left(-\frac{u^{\prime}}{r^{2}}-\frac{2}{r^{2}} \frac{\partial v^{\prime}}{\partial \theta}\right) .
\end{aligned}
$$

\section{Appendix B. Accuracy of numerical simulation}

In this section we check the accuracy of DNS by showing dependence on the spatial resolution, the radial domain size, the time step and the relaxation time of the base flow. We choose the curvature instability mode of $(-1,0 ;[1,3])$ with $\varepsilon_{0}=0.1$ and $k=1.144$. Table B compares the growth rate obtained for different numerical settings. In this table 'reference' is used in the results of the present paper. In case G, the minimum/constant grid size is doubled in the $r / z$ direction, while the time step is also doubled to keep the 
Courant number constant. The growth rate is unchanged up to the digits shown in the table, showing that the grid resolution is sufficient and the coarser grid of case G could have been used. Having confirmed grid resolution, dependence on the radial domain size $L_{r}$ is checked with cases L1, L2 and L3 with $L_{r} / R_{0}=50,25$ and 10. Again the growth rate is unchanged up to the digits shown in the table. By halving the time step (case DT) from case $\mathrm{G}$ the growth rate decreases a little with $0.015 \%$ change, which is sufficiently small.

In case TL, we changed the relaxation time of the base flow to $T_{0}=24$, while the results shown in the present paper are based on $T_{0}=12$ and 16 for $R e=10^{4}$ and $5 \times 10^{4}$, respectively. The growth rate decreases a little with $0.75 \%$ change, which is also sufficiently small for the purpose of the present paper.

\section{REFERENCES}

Blanco-Rodríguez, F. J. \& Le Dizès, S., Selçuk, C., Delbende, I. \& Rossi, M. 2015 Internal structure of vortex rings and helical vortices. J. Fluid Mech. 785, 219-247.

Blanco-Rodríguez, F. J. \& Le Dizès, S. 2016 Elliptic instability of a curved Batchelor vortex. J. Fluid Mech. 804, 224-247.

Blanco-Rodríguez, F. J. \& Le Dizès, S. 2017 Curvature instability of a curved Batchelor vortex. J. Fluid Mech. 814, 397-415.

Dazin, A., Dupont, P. \& Stanislas, M. 2006a Experimental characterization of the instability of the vortex ring. Part I: Linear phase. Exp. Fluids 40, 383-399.

Dazin, A., Dupont, P. \& Stanislas, M. 2006b Experimental characterization of the instability of the vortex rings. Part II: Non-linear phase. Exp. Fluids 41, 401-413.

Delbende, I., Rossi, M. \& Daube, O. 2012 DNS of flows with helical symmetry. Theor. Comput. Fluid Dyn. 26, 141-160.

Eloy, C., Le Gal, P. \& Le Diż̀s, S. 2000 Experimental Study of the Multipolar Vortex Instability. Phys. Rev. Lett. 85, 3000-3003.

Eloy, C., Le Gal, P. \& Le DizÈs, S. 2003 Elliptic and triangular instabilities in rotating cylinders. J. Fluid Mech. 476, 357-388.

Fabre, D., Sipp, D. \& Jacquin, L. 2006 Kelvin waves and the singular modes of the LambOseen vortex. J. Fluid Mech. 551, 235-274.

Fukumoto, Y. \& Hattori, Y. 2005 Curvature instability of a vortex ring. J. Fluid Mech. 526, $77-115$.

Fukumoto, Y. \& Moffatt, H. K. 2000 Motion and expansion of a viscous vortex ring. Part 1. A higher-order asymptotic formula for the velocity. J. Fluid Mech. 417, 1-45.

Gan, L. Nickels, T. B. \& Dawson, J. R. 2011 An experimental study of a turbulent vortex ring: a three-dimensional representation. Exp Fluids 51, 1493-1507.

Gargan-Shingles, C., Rudman, M. \& Ryan, K. 2016 The linear stability of swirling vortex rings. Phys. Fluids 28, 114106.

Hattori, Y. \& Fukumoto, Y. 2003 Short-wavelength stability analysis of thin vortex rings. Phys. Fluids 15, 3151-3163.

Hattori, Y. \& Fukumoto, Y. 2009 Short-wavelength stability analysis of a helical vortex tube. Phys. Fluids 21, 014104.

Hattori, Y. \& Fukumoto, Y. 2010 Short-wave stability of a helical vortex tube: the effect of torsion on the curvature instability. Theor. Comput. Fluid Dyn. 24, 363-368.

Hattori, Y. \& Fukumoto, Y. 2011 Erratum: Short-wavelength stability analysis of a helical vortex tube. Phys. Fluids 23, 049901.

Hattori, Y. \& Fukumoto, Y. 2012 Effects of axial flow on the stability of a helical vortex tube. Phys. Fluids 24, 054102.

Hattori, Y. \& Fukumoto, Y. 2014 Modal stability analysis of a helical vortex tube with axial flow. J. Fluid Mech. 738, 222-249.

HAtToRi, Y. \& HiJiYA, K. 2010 Short-wavelength stability analysis of Hill's vortex with/without swirl. Phys. Fluids 22, 074104. 
Kerswell, R. 1993 The instability of precessing flow. Geophys. Astrophys. Fluid. Dyn. 72, $107-144$.

Knobloch, E., Mahalov, A. \& Marsden, J. E. 1994 Normal forms for three-dimensional parametric instabilities in ideal hydrodynamics. Physica D $\mathbf{7 3} 49-81$.

Lacaze L., Birbaud, A.-L. \& Le Dizès, S. 2005 Elliptic instability in a Rankine vortex with axial flow. Phys. Fluids 17, 017101.

Lacaze, L., Ryan, K. \& Le DizÈs, S. 2007 Elliptic instability in a strained Batchelor vortex. J. Fluid Mech. 577, 341-361.

Lagrange, R., Eloy, C., Nadal, F. \& Meunier, P. 2008 Instability of a fluid inside a precessing cylinder. Phys. Fluids 20, 081701.

LE Dizès, S. 2004 Viscous critical-layer analysis of vortex normal modes. Stud. Appl. Maths. 112, 315-332.

LeLe, S. K. 1992 Compact finite-difference schemes with spectral-like resolution. J. Comput. Phys. 103, 16-42.

Mahalov, A. 1993 The instability of rotating fluid columns subjected to a weak external Coriolis force. Phys. Fluids A 5, 891-900.

Mao, X. \& Hussain, F. 2017 Optimal transient growth on a vortex ring and its transition via cascade of ringlets. J. Fluid Mech. 832, 269-286.

Maxworthy, T. 1972 The structure and stability of vortex rings. J. Fluid Mech. 51, 15-32.

Maxworthy, T. 1977 Some experimental studies of vortex rings. J. Fluid Mech. 81, 465-495.

Moore, D. W. \& Saffman, P. G. 1972 The motion of a vortex filament with axial flow. Phil. Trans. R. Soc. Lond. A 272, 403-429.

Moore, D. W. \& Saffman, P. G. 1975 The instability of a straight vortex filament in a strain field. Proc. R. Soc. Lond. A 346, 413-425.

Roy, C., Schaeffer, N., Le Dizès, S. \& Thompson, M. 2008 Phys. Fluids 20, 094101.

Saffman, P. G. 1992 Vortex dynamics, Chap. 2, Chap. 10. Cambridge University Press.

Selçuk, C., Delbende, I. \& Rossi, M. 2018 Helical vortices: linear stability analysis and nonlinear dynamics. Fluid Dyn. Res. 50, 011411.

Shariff, K., Verzicco, R. \& Orlandi, P. 1994 A numerical study of three-dimensional vortex ring instabilities: viscous corrections and early nonlinear stage. J. Fluid Mech. 279, 351-375.

SiPP, D. 2000 Weakly nonlinear saturation of short-wave instabilities in a strained Lamb-Oseen vortex. Phys. Fluids 12, 1715-1729.

Thompson, M. C., Leweke, T. \& Hourigan, K. 2007 Spherewall collisions: vortex dynamics and stability. J. Fluid Mech. 575, 121-148.

Widnall, S. E. \& Tsai, C.-Y. 1976 The stability of short waves on a straight vortex filament in a weak externally imposed strain field. J. Fluid Mech. 73, 721-733.

Widnall, S. E. 1972 The stability of a helical vortex filament. J. Fluid Mech. 54, 641-663.

Widnall, S. E. Bliss, D. B. \& ZALAY, Y. 1971 Theoretical and experimental study of the stability of a vortex pair. In Aircraft Wake Turbulence and Its Detection (ed. J. H. Olsen, A. Goldburg \& M. Rogers), pp. 305-338. Springer.

Widnall, S. E. \& Sullivan, J. P. 1973 On the stability of vortex rings. Proc. R. Soc. Lond. A 332, 335-353.

WidnalL, S. E. \& TSAI, C.-Y. 1977 The instability of the thin vortex ring of constant vorticity. Phil. Trans. R. Soc. Lond. A 287, 273-305. 